\title{
Multiple boundary peak solutions for some singularly perturbed Neumann problems
}

by

\author{
Changfeng GUI ${ }^{\mathrm{a}, 1}$, Juncheng WEI ${ }^{\mathrm{b}, 2}$, Matthias WINTER ${ }^{\mathrm{c}, 3}$ \\ ${ }^{a}$ Department of Mathematics, University of British Columbia, \\ Vancouver, BC V6T 1Z2, Canada \\ $\mathrm{b}$ Department of Mathematics, The Chinese University of Hong Kong, \\ Shatin, Hong Kong \\ c Mathematisches Institut A, Universität Stuttgart, D-70511 Stuttgart, Germany \\ Manuscript received 1 October 1997, revised 18 July 1998
}

ABSTRACT. - We consider the problem

$$
\left\{\begin{array}{l}
\varepsilon^{2} \Delta u-u+f(u)=0 \quad \text { in } \Omega, \\
u>0 \quad \text { in } \Omega, \quad \partial u / \partial v=0 \quad \text { on } \partial \Omega,
\end{array}\right.
$$

where $\Omega$ is a bounded smooth domain in $R^{N}, \varepsilon>0$ is a small parameter and $f$ is a superlinear, subcritical nonlinearity. It is known that this equation possesses boundary spike solutions such that the spike concentrates, as $\varepsilon$ approaches zero, at a critical point of the mean curvature function $H(P), P \in \partial \Omega$. It is also known that this equation has multiple boundary spike solutions at multiple nondegenerate critical points of $H(P)$ or multiple local maximum points of $H(P)$.

In this paper, we prove that for any fixed positive integer $K$ there exist boundary $K$-peak solutions at a local minimum point of $H(P)$. This implies that for any smooth and bounded domain there always exist boundary $K$-peak solutions.

\footnotetext{
${ }^{1}$ E-mail: cgui@ math.ubc.ca. Current address: Department of Mathematics, University of Connecticut, Storrs, CT 06269, USA.

${ }^{2}$ E-mail: wei@math.cuhk.edu.hk.

${ }^{3}$ E-mail: winter@mathematik.uni-stuttgart.de.
} 
We first use the Liapunov-Schmidt method to reduce the problem to finite dimensions. Then we use a maximizing procedure to obtain multiple boundary spikes.

(C) 2000 L'Association Publications de l'Institut Henri Poincaré. Published by Elsevier B.V. All rights reserved

Key words: Multiple boundary spikes, nonlinear elliptic equations

RÉSUMÉ. - Nous considerons le problème

$$
\left\{\begin{array}{l}
\varepsilon^{2} \Delta u-u+f(u)=0 \quad \text { in } \Omega, \\
u>0 \quad \text { in } \Omega, \quad \partial u / \partial v=0 \quad \text { on } \partial \Omega,
\end{array}\right.
$$

où $\Omega$ est une domaine bornée avec frontiére lisse en $R^{N}, \varepsilon>0$ est un parametre petit, et $f$ est surlinéaire et souscritique. Il est bien connu que cette équation possede des solutions avec pointe sur la frontiére telle que la pointe se concentre (quand $\varepsilon$ tend vers zero) à une pointe critique de la courbure moyenne $H(P), P \in \partial \Omega$. Il est aussi connu que cette équation possede pleusieurs solutions avec pointes qui se concentrent sur pleusieurs points critiques nondégénerés de $H(P)$, ou sur pleusieurs maxima locaux de $H(P)$.

Dans ce papier, nous prouvons que, pour chaque entier positif $K$ donné, il existe solutions avec $K$ pointes l̀a frontiére, situées sur un minimum relatif de $H(P)$. Ceci implique que pour chaque domaine qui est lisse et bornée il existe toujours des solutions avec $K$ pointes à la frontiére.

Nous utilisons la methode de Liapunov-Schmidt pour reduire le problème dans une espace de dimension finie. Ensuite, nous utilisons une procédé de maximization pour obtenir les pointes sur la frontiére.

(C) 2000 L'Association Publications de l'Institut Henri Poincaré. Published by Elsevier B.V. All rights reserved

\section{INTRODUCTION}

The aim of this paper is to construct a family of multiple boundary peak solutions to the following singularly perturbed elliptic problem

$$
\left\{\begin{array}{l}
\varepsilon^{2} \Delta u-u+u^{p}=0 \quad \text { in } \quad \Omega \\
u>0 \quad \text { in } \Omega \quad \text { and } \quad \partial u / \partial v=0 \quad \text { on } \partial \Omega
\end{array}\right.
$$


where $\Delta=\sum_{i=1}^{N}\left(\partial^{2} / \partial x_{i}^{2}\right)$ is the Laplace operator, $\Omega$ is a bounded smooth domain in $R^{N}, \varepsilon>0$ is a constant, the exponent $p$ satisfies $1<p<(N+2) /(N-2)$ for $N \geqslant 3$ and $1<p<\infty$ for $N=2$ and $v(x)$ denotes the normal derivative at $x \in \partial \Omega$.

Eq. (1.1) is known as the stationary equation of the Keller-Segal system in chemotaxis. It can also be seen as the limiting stationary equation of the so-called Gierer-Meinhardt system in biological pattern formation, see [35] for more details.

In the pioneering papers of [18,21] and [22], Lin, Ni and Takagi established the existence of least-energy solutions and showed that for $\varepsilon$ sufficiently small the least-energy solution has only one local maximum point $P_{\varepsilon}$ and $P_{\varepsilon} \in \partial \Omega$. Moreover, $H\left(P_{\varepsilon}\right) \rightarrow \max _{P \in \partial \Omega} H(P)$ as $\varepsilon \rightarrow 0$, where $H(P)$ is the mean curvature of $P$ at $\partial \Omega$. In [23], Ni and Takagi constructed boundary spike solutions for axially symmetric domains. The second author in [35] studied the general domain case and showed that for single boundary spike solutions, the boundary spike must approach a critical point of the mean curvature; on the other hand, for any nondegenerate critical point of $H(P)$, one can construct boundary spike solutions whose spike approaches that point. The first author in [11] constructed multiple boundary spike layer solutions at multiple local maximum points of $H(P)$. Later the second and third authors in [38] constructed multiple boundary spike layer solutions at multiple nondegenerate critical points of $H(P)$. Related results were obtained independently by Y.Y. Li in [17]. When $p=(N+2) /(N-2)$, similar results for the boundary spike layer solutions have been obtained in $[1-3,12,20,27-29,31]$, etc. We also note that multiple interior peak solutions in general domain are obtained in [13].

In this paper, we study the existence of multiple boundary peak solutions at a local minimum point of $H(P)$.

More precisely, we consider the problem

$$
\left\{\begin{array}{l}
\varepsilon^{2} \Delta u-u+f(u)=0 \quad \text { in } \Omega, \\
u>0 \text { in } \Omega \text { and } \partial u / \partial v=0 \text { in } \Omega .
\end{array}\right.
$$

We will assume that $f: R^{+} \rightarrow R$ is of class $C^{1+\sigma}$ and satisfies the following conditions

(f1) $f(t) \equiv 0$ for $t \leqslant 0$ and $f(t) \rightarrow+\infty$ as $t \rightarrow \infty$. 
(f2) There exist some constants $1<p_{1}, p_{2}, p_{3}<((N+4) /(N-$ $4))_{+}(=\infty$ if $N \leqslant 4 ;=(N+4) /(N-4)$ if $N>4)$ such that $f(0)=0, f^{\prime}(0)=0$ and

$$
\begin{aligned}
& f(u)=\mathrm{O}\left(|u|^{p_{1}}\right), \quad f^{\prime}(u)=\mathrm{O}\left(|u|^{p_{2}-1}\right) \quad \text { as }|u| \rightarrow \infty, \\
& \left|f_{u}(u+\phi)-f_{u}(u)\right| \leqslant \begin{cases}C|\phi|^{p_{3}-1} & \text { if } p_{3}>2, \\
C\left(|\phi|+|\phi|^{p_{3}-1}\right) & \text { if } p_{3} \leqslant 2 .\end{cases}
\end{aligned}
$$

(f3) The equation

$$
\left\{\begin{array}{l}
\Delta w-w+f(w)=0 \quad \text { in } R^{N} \\
w>0, \quad w(0)=\max _{z \in R^{N}} w(z) \\
w \rightarrow 0 \quad \text { at } \infty
\end{array}\right.
$$

has a unique solution $w(y)$ (by the results of [9], $w$ is radial, i.e., $w=w(r)$ and $w^{\prime}<0$ for $\left.r=|y| \neq 0\right)$ and $w$ is nondegenerate. Namely the operator

$$
L:=\Delta-1+f^{\prime}(w)
$$

is invertible in the space $H_{r}^{2}\left(R^{N}\right):=\left\{u=u(|y|) \in H^{2}\left(R^{N}\right)\right\}$.

Two important examples of $f$ are the following.

Example 1 (Chemotaxis and pattern formation). $-f(u)=u^{p}$ where $1<p<((N+2) /(N-2))_{+}(=\infty$ if $N=2 ;=(N+2) /(N-2)$ if $N>$ 2 ). It is easy to see that $f$ satisfies (f1), (f2) and (f3). This problem arises from the Keller-Segal model in chemotaxis and the Gierer-Meinhardt system in pattern formation (see $[21,22]$ and the references therein).

Example 2 (Population dynamics and chemical reaction theory). -

$$
f(u)=u(u-a)(1-u)
$$

where $0<a<1 / 2$. This is a famous model from population dynamics and chemical reaction theory (see $[5,15,30]$ ). If $N \leqslant 8$ then by the result of [8], $f$ satisfies (f1)-(f3).

Other nonlinearities satisfying (f1), (f2) and (f3) can be found in [6].

Let $\Lambda \subset \partial \Omega$ be an open set such that

$$
\min _{P \in \partial \Gamma} H(P)>\min _{P \in \Gamma} H(P) .
$$


We now state the main result in this paper.

THEOREM 1.1. - Assume that condition (1.5) holds. Let $f$ satisfy assumptions (f1)-(f3). Then for $\varepsilon$ sufficiently small problem (1.2) has a solution $u_{\varepsilon}$ which possesses exactly $K$ local maximum points $Q_{1}^{\varepsilon}, \ldots, Q_{K}^{\varepsilon}$ with $\mathbf{Q}^{\varepsilon}=\left(Q_{1}^{\varepsilon}, \ldots, Q_{K}^{\varepsilon}\right) \in \Gamma \times \cdots \times \Gamma$. Moreover

$$
H\left(Q_{i}^{\varepsilon}\right) \rightarrow \min _{P \in \Gamma} H(P), \quad w\left(\frac{\left|Q_{k}^{\varepsilon}-Q_{l}^{\varepsilon}\right|}{\varepsilon}\right) \rightarrow 0,
$$

$i, k, l=1, \ldots, K, k \neq l$, as $\varepsilon \rightarrow 0$. Furthermore, we have

$$
u_{\varepsilon}(x) \leqslant a \exp \left(-\frac{b \min _{i=1, \ldots, K}\left(\left|x-Q_{i}^{\varepsilon}\right|\right)}{\varepsilon}\right)
$$

for certain positive constants $a, b$.

Theorem 1.1 can be derived from a more general theorem as follows.

THEOREM 1.2. - Let $\Gamma_{i}, i=1, \ldots, K$, be open sets in $\partial \Omega$ such that

$$
\min _{P \in \partial \Gamma_{i}} H(P)>\min _{P \in \Gamma_{i}} H(P), \quad i=1, \ldots, K
$$

Let $f$ satisfy assumptions (f1)-(f3). Then for $\varepsilon$ sufficiently small problem (1.2) has a solution $u_{\varepsilon}$ which possesses exactly $K$ local maximum points $Q_{1}^{\varepsilon}, \ldots, Q_{K}^{\varepsilon}$ with $\mathbf{Q}^{\varepsilon}=\left(Q_{1}^{\varepsilon}, \ldots, Q_{K}^{\varepsilon}\right) \in \Gamma_{1} \times \cdots \times \Gamma_{K}$. Moreover

$$
H\left(Q_{i}^{\varepsilon}\right) \rightarrow \min _{P \in \Gamma_{i}} H(P), \quad w\left(\frac{\left|Q_{k}^{\varepsilon}-Q_{l}^{\varepsilon}\right|}{\varepsilon}\right) \rightarrow 0,
$$

$i, k, l=1, \ldots, K, k \neq l$, as $\varepsilon \rightarrow 0$. Furthermore, we have

$$
u_{\varepsilon}(x) \leqslant a \exp \left(-\frac{b \min _{i=1, \ldots, K}\left(\left|x-Q_{i}^{\varepsilon}\right|\right)}{\varepsilon}\right)
$$

for certain positive constants $a, b$.

More details about the asymptotic behavior of $u_{\varepsilon}$ can be found in the proof of Theorem 1.2.

We have the following interesting corollary.

COROLlARY 1.3. - For any smooth and bounded domain and any fixed positive integer $K \in Z$, there always exists a boundary $K$-peaked solution of (1.2) if $\varepsilon$ is small enough. 
Theorem 1.1 is the first result in proving the existence of multiple boundary spike solutions for problem (1.2) in any smooth bounded domain. Note that the boundary spikes can approach the same point on the boundary when $\Lambda$ has a strictly local minimum point of $H(P)$. This is new and interesting in its own right.

We shall only prove Theorem 1.2. To introduce the main idea of the proof of Theorem 1.2, we need to give some necessary notations and definitions first.

Let $w$ be the unique solution of (1.3). It is known (see [9]) that $w$ is radially symmetric, decreasing and

$$
\lim _{|y| \rightarrow \infty} w(y) \mathrm{e}^{|y|}|y|^{\frac{N-1}{2}}=c_{0}>0 .
$$

Associated with problem (1.2) is the following energy functional

$$
J_{\varepsilon}(u)=\frac{1}{2} \int_{\Omega}\left(\varepsilon^{2}|\nabla u|^{2}+u^{2}\right)-\int_{\Omega} F(u)
$$

where $F(u)=\int_{0}^{u} f(s) \mathrm{d} s$ and $u \in H^{1}(\Omega)$.

For any smooth bounded domain $U$ we set $P_{U} w$ to be the unique solution of

$$
\begin{cases}\Delta u-u+f(w)=0 & \text { in } U, \\ \partial u / \partial v=0 & \text { on } U .\end{cases}
$$

Let $\eta>0$ be a small number. Let $\Gamma_{i}$ be as in Theorem 1.2. Set

$$
\begin{gathered}
\Lambda=\left\{\mathbf{P}=\left(P_{1}, \ldots, P_{K}\right) \in \Gamma_{1} \times \cdots \times \Gamma_{K}, w\left(\frac{\left|P_{k}-P_{l}\right|}{\varepsilon}\right)<\eta \varepsilon,\right. \\
k, l=1, \ldots, K, k \neq l\} .
\end{gathered}
$$

For $P \in \partial \Omega$, we set

$$
\Omega_{\varepsilon}=\{y: \varepsilon y \in \Omega\}, \quad \Omega_{\varepsilon, P}=\{y: \varepsilon y+P \in \Omega\} .
$$

Fix $\mathbf{P}=\left(P_{1}, P_{2}, \ldots, P_{K}\right) \in \bar{\Lambda}$. We set

$$
\begin{gathered}
P w_{i}(y)=P_{\Omega_{\varepsilon, P_{i}}} w\left(y-\frac{P_{i}}{\varepsilon}\right), \quad w_{i}(y)=w\left(y-\frac{P_{i}}{\varepsilon}\right), \quad y \in \Omega_{\varepsilon}, \\
u=\sum_{i=1}^{K} P w_{i}+\Phi_{\varepsilon, \mathbf{P}} \in H^{2}\left(\Omega_{\varepsilon}\right),
\end{gathered}
$$




$$
\begin{aligned}
& \mathcal{K}_{\varepsilon, \mathbf{P}}=\operatorname{span}\left\{\frac{\partial P w_{i}}{\partial \tau_{P_{i, i}}}, i=1, \ldots, K, j=1, \ldots, N-1\right\} \subset H^{2}\left(\Omega_{\varepsilon}\right), \\
& \mathcal{C}_{\varepsilon, \mathbf{P}}=\operatorname{span}\left\{\frac{\partial P w_{i}}{\partial \tau_{P_{i, i}}}, i=1, \ldots, K, j=1, \ldots, N-1\right\} \subset L^{2}\left(\Omega_{\varepsilon}\right),
\end{aligned}
$$

where $\tau_{P_{i, i_{j}}}$ are the $(N-1)$ tangential derivatives at $P_{i}$ (without loss of generality we assume that the inward normal derivative at $P_{i}$ is $e_{N}$ and denote $\tau_{P_{i, i} j}$ as $\tau_{P_{i, j}}$ in the rest of the paper.)

We first solve for $\Phi_{\varepsilon, \mathbf{P}}$ in $\mathcal{K}_{\varepsilon, \mathbf{P}}^{\perp}$ up to $\mathcal{C}_{\varepsilon, P}^{\perp}$ by using the LiapunovSchmidt reduction method. This method evolves from that of [7,25] and [26] on the semi-classical (i.e., for small parameter $h$ ) solution of the nonlinear Schrödinger equation

$$
\frac{h^{2}}{2} \Delta U-(V-E) U+U^{p}=0
$$

in $R^{N}$ where $V$ is a potential function and $E$ is a real constant. The method of Liapunov-Schmidt reduction was used in [7,25] and [26] to construct solutions of (1.9) close to nondegenerate critical points of $V$ for $h$ sufficiently small.

Then we show that $\Phi_{\varepsilon, \mathbf{P}}$ is $C^{1}$ in $\mathbf{P}$. After that, we define a new function

$$
M_{\varepsilon}(\mathbf{P})=J_{\varepsilon}\left(\sum_{i=1}^{K} P w_{i}+\Phi_{\varepsilon, \mathbf{P}}\right)
$$

We maximize $M_{\varepsilon}(\mathbf{P})$ over $\bar{\Lambda}$. Condition (1.5) ensures that $M_{\varepsilon}(\mathbf{P})$ attains its maximum inside $\Lambda$. We show that the resulting solution has the properties of Theorem 1.2.

The paper is organized as follows. Notation, preliminaries and some useful estimates are explained in Section 2. Section 3 contains the setup of our problem and we solve (1.2) up to approximate kernel and cokernel, respectively. We set up and solve a maximizing problem in Section 4. Finally, in Section 5, we show that the solution to the maximizing problem is indeed a solution of (1.2) and satisfies all the properties of Theorem 1.2.

Throughout this paper, unless otherwise stated, the letter $C$ will always denote various generic constants which are independent of $\varepsilon$, for $\varepsilon$ sufficiently small. $\delta>0$ is a very small number. o(1) means $|\mathrm{o}(1)| \rightarrow 0$ as $\varepsilon \rightarrow 0$. 


\section{TECHNICAL ANALYSIS}

In this section we introduce a projection and derive some useful estimates. Throughout the paper we shall use the letter $C$ to denote a generic positive constant which may vary from term to term. We denote $R_{+}^{N}=\left\{\left(x^{\prime}, x_{N}\right) \mid x_{N}>0\right\}$. Let $w$ be the unique solution of (1.3).

Set

$$
I(w)=\frac{1}{2} \int_{R^{N}}\left(|\nabla w|^{2}+w^{2}\right)-\int_{R^{N}} F(w) .
$$

Let $P \in \partial \Omega$. We can define a diffeomorphism straightening the boundary in a neighborhood of $P$. After rotation of the coordinate system we may assume that the inward normal to $\partial \Omega$ at $P$ is pointing in the direction of the positive $x_{N}$-axis. Denote $x^{\prime}=\left(x_{1}, \ldots, x_{N-1}\right)$,

$$
\begin{gathered}
B^{\prime}\left(R_{0}\right)=\left\{x^{\prime} \in R^{N-1}|| x^{\prime} \mid<R_{0}\right\}, \\
B\left(P, R_{0}\right)=\left\{x \in R^{N}|| x-P \mid<R_{0}\right\},
\end{gathered}
$$

and

$$
\begin{aligned}
\Omega_{0} & =\Omega \cap B\left(P, R_{0}\right) \\
& =\left\{\left(x^{\prime}, x_{N}\right) \in B\left(P, R_{0}\right) \mid x_{N}-P_{N}>\rho\left(x^{\prime}-P^{\prime}\right)\right\} .
\end{aligned}
$$

Then, since $\partial \Omega$ is smooth, we can find a constant $R_{0}>0$ such that $\partial \Omega \cap$ $\overline{\Omega_{0}}$ can be represented by the graph of a smooth function $\rho_{P}: B^{\prime}\left(R_{0}\right) \rightarrow$ $R$ where $\rho_{P}(0)=0, \nabla \rho_{P}(0)=0$.

From now on we omit the use of $P$ in $\rho_{P}$ and write $\rho$ instead if this can be done without causing confusion. The mean curvatures of $\partial \Omega$ at $P$ is

$$
H(P)=\frac{1}{n-1} \sum_{i=1}^{N-1} \rho_{i i}(0),
$$

where

$$
\rho_{i}=\frac{\partial \rho}{\partial x_{i}}, \quad i=1, \ldots, N-1,
$$

and higher derivatives are defined in the same way. By Taylor expansion we have

$$
\rho\left(x^{\prime}-P^{\prime}\right)=\frac{1}{2} \sum_{i, j=1}^{N-1} \rho_{i j}(0)\left(x_{i}-P_{i}\right)\left(x_{j}-P_{j}\right)
$$




$$
\begin{aligned}
& +\frac{1}{6} \sum_{i, j, k=1}^{N-1} \rho_{i j k}(0)\left(x_{i}-P_{i}\right)\left(x_{j}-P_{j}\right)\left(x_{k}-P_{k}\right) \\
& +\mathrm{O}\left(\left|x^{\prime}-P^{\prime}\right|^{4}\right) .
\end{aligned}
$$

Recall that for a smooth bounded domain $U$ the projection $P_{U}$ of $H^{2}(U)$ onto $\left\{v \in H^{2}(U) \mid \partial v / \partial v=0\right.$ at $\left.\partial U\right\}$ is defined as follows: For $v \in H^{2}(U)$ let $\omega=P_{U} v$ be the unique solution of the boundary value problem

$$
\begin{cases}\Delta \omega-\omega+f(v)=0 & \text { in } U \\ \partial \omega / \partial v=0 & \text { on } \partial U\end{cases}
$$

Let

$$
h_{\varepsilon, P}(x)=w\left(\frac{x-P}{\varepsilon}\right)-P_{\Omega_{\varepsilon, P}} w\left(\frac{x-P}{\varepsilon}\right), \quad x \in \Omega,
$$

where

$$
\Omega_{\varepsilon, P}=\left\{z \in R^{n} \mid \varepsilon z+P \in \Omega\right\} .
$$

Then $h_{\varepsilon, P}$ satisfies

$$
\begin{cases}\varepsilon^{2} \Delta v-v=0 & \text { in } \Omega \\ \partial v / \partial v=(\partial / \partial v) w((x-P) / \varepsilon) & \text { on } \partial \Omega\end{cases}
$$

We denote

$$
\|v\|_{\varepsilon}^{2}=\varepsilon^{-N} \int_{\Omega}\left[\varepsilon^{2}|\nabla v|^{2}+v^{2}\right] .
$$

For $x \in \Omega_{0}$ set now

$$
\left\{\begin{array}{l}
\varepsilon y^{\prime}=x^{\prime}-P^{\prime} \\
\varepsilon y_{N}=x_{N}-P_{N}-\rho\left(x^{\prime}-P^{\prime}\right)
\end{array}\right.
$$

Furthermore, for $x \in \Omega_{0}$ we introduce the transformation $T$ by

$$
\left\{\begin{array}{l}
T_{i}\left(x^{\prime}\right)=x_{i}, \quad i=1, \ldots, N-1 \\
T_{N}\left(x^{\prime}\right)=x_{N}-P_{N}-\rho\left(x^{\prime}-P^{\prime}\right) .
\end{array}\right.
$$


Note that then

$$
y=\frac{1}{\varepsilon} T(x) .
$$

Let $v_{1}$ be the unique solution of

$$
\begin{cases}\Delta v-v=0 & \text { in } R_{+}^{N}, \\ \frac{\partial v}{\partial y_{N}}=-\frac{w^{\prime}}{|y|} \frac{1}{2} \sum_{i, j=1}^{N-1} \rho_{i j}(0) y_{i} y_{j} & \text { on } \partial R_{+}^{N},\end{cases}
$$

where $w^{\prime}$ is the radial derivative of $w$, i.e., $w^{\prime}=w_{r}(r)$, and $r=\mid(x-$ $P) / \varepsilon \mid$.

Note that $v_{1}$ is an even functions in $y^{\prime}=\left(y_{1}, \ldots, y_{N-1}\right)$. Moreover, it is easy to see that $\left|v_{1}\right| \leqslant C \mathrm{e}^{-\mu|y|}$ for some $0<\mu<1$.

Let $\chi(x)$ be a smooth cut-off function such that $\chi(x)=1, x \in$ $B\left(0,0.8 R_{0}\right)$ and $\chi(x)=0$ for $x \in B\left(0, R_{0}\right)^{C}$.

In fact we set $R_{0}$ be such that $w\left(R_{0} / \varepsilon\right)=0.9 \eta \varepsilon$.

Note that this $\chi$ is as good as the cut-off function in [35].

Set

$$
h_{\varepsilon, P}(x)=\varepsilon v_{1}(y) \chi(x-P)+\varepsilon^{2} \Psi_{\varepsilon, P}(x), \quad x \in \Omega .
$$

Then we have

PROPOSITION 2.1. -

$$
\left\|\Psi_{\varepsilon, P}\right\|_{\varepsilon} \leqslant C \text {. }
$$

Proof. - Proposition 2.1 was proved in [37] by Taylor expansion and a rigorous estimate for the remainder using estimates for elliptic partial differential equations.

Similarly, we know from [37] that

PROPOSITION 2.2.-

$$
\left[\frac{\partial w}{\partial \tau_{P_{j}}}-\frac{\partial P_{\Omega_{\varepsilon, P}} w}{\partial \tau_{P_{j}}}\right]\left(\frac{x-P}{\varepsilon}\right)=w_{1}(y) \chi(x-P)+\varepsilon w_{2}^{\varepsilon}(x), \quad x \in \Omega,
$$

where $\varepsilon y=T(x)$ and $w_{1}$ satisfies 


$$
\left\{\begin{array}{rlrl}
\Delta v-v & =0 & & \text { in } R_{+}^{N} \\
\frac{\partial v}{\partial y_{N}}= & -\frac{1}{2}\left(\frac{w^{\prime \prime}}{|y|^{2}}-\frac{w^{\prime}}{|y|^{3}}\right) \sum_{k, l=1}^{N-1} \rho_{k l}(0) y_{k} y_{l} y_{j} & \\
& -\frac{w^{\prime}}{|y|} \sum_{k=1}^{N-1} \rho_{j k}(0) y_{k} & & \text { on } \partial R_{+}^{N}
\end{array}\right.
$$

and $\left\|w_{2}^{\varepsilon}\right\|_{\varepsilon} \leqslant C$.

Note that $\left|w_{1}\right| \leqslant C \exp (-\mu|y|)$ for some $\mu<1$ and $w_{1}$ is an odd function in $y^{\prime}$. Finally, let $L_{0}=\Delta-1+f^{\prime}(w)$. We have

LEMMA 2.3. -

$$
\operatorname{Ker}\left(L_{0}\right) \cap H_{N}^{2}\left(R_{+}^{N}\right)=\operatorname{span}\left\{\frac{\partial w}{\partial y_{1}}, \ldots, \frac{\partial w}{\partial y_{N-1}}\right\},
$$

where $H_{N}^{2}\left(R_{+}^{N}\right)=\left\{u \in H^{2}\left(R_{+}^{N}\right), \partial u / \partial y_{N}=0\right.$ on $\left.\partial R_{+}^{N}\right\}$.

Proof. - See Lemma 4.2 in [22].

Next we state some useful lemmas about the interactions of two w's.

LemMA 2.4. - Let $\mathbf{P}=\left(P_{1}, \ldots, P_{K}\right) \in \bar{\Lambda}$. Then we have

$$
\int_{\Omega_{\varepsilon}} f\left(w_{k}\right) w_{l}=\left(\gamma_{k l}+\mathrm{o}(1)\right) w\left(\frac{\left|P_{k}-P_{l}\right|}{\varepsilon}\right), \quad k, l=1, \ldots, K, k \neq l,
$$

where $\gamma_{k l} \in \Sigma$ and $\Sigma$ is defined as follows

$$
\Sigma=\left\{\int_{R_{+}^{N}} f(w(y)) \mathrm{e}^{\langle b, y\rangle} \mathrm{d} y\left|b \in R^{N},\right| b \mid=1\right\} .
$$

Furthermore, if $w\left(\left|P_{k}-P_{l}\right| / \varepsilon\right)=\eta \varepsilon$, we have $\gamma_{k l} \in \Sigma_{1}$ where

$$
\begin{gathered}
\Sigma_{1}=\left\{\int_{R_{+}^{N}} f(w(y)) \mathrm{e}^{\langle b, y\rangle} \mathrm{d} y \mid b=\left(b_{1}, \ldots, b_{N}\right) \in R^{N},\right. \\
\left.b_{N}=0,|b|=1\right\} .
\end{gathered}
$$

Proof. - Note that as $\left|P_{k}-P_{l}\right| / \varepsilon \rightarrow \infty$ we have

$$
w\left(\frac{\left|P_{k}-P_{l}\right|}{\varepsilon}\right)=\left(\frac{\left|P_{k}-P_{l}\right|}{\varepsilon}\right)^{-\frac{N-1}{2}} \mathrm{e}^{-\frac{\left|P_{k}-P_{l}\right|}{\varepsilon}}(1+\mathrm{o}(1)) .
$$


Hence if we straighten the boundary at $P_{k}$ we have

$$
\begin{aligned}
\int_{\Omega_{\varepsilon}} f\left(w_{k}\right) w_{l}= & \int_{R_{+}^{N}} f(w(y)) w\left(y-\frac{P_{l}-P_{k}}{\varepsilon}\right)(1+\mathrm{o}(1)) \\
= & w\left(\frac{\left|P_{k}-P_{l}\right|}{\varepsilon}\right)(1+\mathrm{o}(1)) \int_{R_{+}^{N}} f(w(y)) \\
& \times w\left(y-\frac{P_{l}-P_{k}}{\varepsilon}\right) w^{-1}\left(\frac{\left|P_{k}-P_{l}\right|}{\varepsilon}\right) \\
= & w\left(\frac{\left|P_{k}-P_{l}\right|}{\varepsilon}\right)(1+\mathrm{o}(1)) \int_{R_{+}^{N}} f(w(y)) \mathrm{e}^{\langle b, y\rangle} \mathrm{d} y
\end{aligned}
$$

for some

$$
b=\lim _{\varepsilon \rightarrow} \frac{P_{k}-P_{l}}{\left|P_{k}-P_{l}\right|} \in R^{N}, \quad|b|=1 .
$$

Note that if $w\left(\left|P_{k}-P_{l}\right| / \varepsilon\right)=\eta \varepsilon$, we have $P_{k} \rightarrow P_{l}$ and $b_{N}=0$ where $b_{N}$ is the $N$ th component of $b$.

Note. $-\gamma_{k l}=\gamma_{l k}$.

Next we are going to show three technical lemmas.

The first lemma is about some relations of several integrals associated with $w$ in $R_{N-1}$.

Let

$$
\gamma_{1}=\frac{1}{N+1} \int_{R^{N-1}}|\nabla w|^{2}\left|y^{\prime}\right|^{2} \mathrm{~d} y^{\prime} .
$$

We have

LEMMA 2.5.-

$$
\begin{aligned}
\frac{N-3}{2} \gamma_{1}= & \int_{R^{N-1}} F\left(w\left(\left|y^{\prime}\right|\right)\right)\left|y^{\prime}\right|^{2} \mathrm{~d} y^{\prime}-\frac{1}{2} \int_{R^{N-1}}|w|^{2}\left|y^{\prime}\right|^{2} \mathrm{~d} y^{\prime} \\
(N+1) \gamma_{1}= & \frac{N-1}{2} \int_{R^{N-1}}|w|^{2} \mathrm{~d} y^{\prime}-\int_{R^{N-1}}|w|^{2}\left|y^{\prime}\right|^{2} \mathrm{~d} y^{\prime} \\
& +\int_{R^{N-1}} f(w) w\left|y^{\prime}\right|^{2} \mathrm{~d} y^{\prime} .
\end{aligned}
$$


Proof. - Let $y=\left(y^{\prime}, y_{N}\right)$. The operators $\Delta$ and $\nabla$ below are with respect to $y \in R^{N}$, and the integrations are with respect to $y^{\prime}=\left(y^{\prime}, 0\right) \in$ $R^{N-1}$. We will also use $r$ for $\left|y^{\prime}\right|$.

By straightforward computations we have

$$
\begin{aligned}
& \int_{R^{N-1}}\left|y^{\prime}\right|^{2} \Delta w(\nabla w \cdot y) \mathrm{d} y^{\prime} \\
& =\omega_{N-2} \int_{0}^{\infty}\left(w^{\prime \prime}(r)+\frac{N-1}{r} w^{\prime}(r)\right) w^{\prime}(r) r^{N+1} \mathrm{~d} r \\
& =\frac{N-3}{2} \omega_{N-2} \int_{0}^{\infty} r^{N}\left(w^{\prime}(r)\right)^{2} \mathrm{~d} r \\
& =\frac{(N+1)(N-3)}{2} \gamma_{1},
\end{aligned}
$$

and

$$
\begin{aligned}
& \int_{R^{N-1}}\left|y^{\prime}\right|^{2} w(\nabla w \cdot y) \mathrm{d} y^{\prime}=\omega_{N-2} \int_{0}^{\infty} w^{\prime}(r) w(r) r^{N+1} \mathrm{~d} r \\
& =-\frac{N+1}{2} \omega_{N-2} \int_{0}^{\infty} r^{N} w^{2} \mathrm{~d} r=-\frac{N+1}{2} \int_{R^{N-1}} w^{2}\left|y^{\prime}\right|^{2} \mathrm{~d} y^{\prime}
\end{aligned}
$$

and

$$
\begin{aligned}
\int_{R^{N-1}}\left|y^{\prime}\right|^{2} f(w)(\nabla w \cdot y) \mathrm{d} y^{\prime} & =\omega_{N-2} \int_{0}^{\infty} f(w) w^{\prime}(r) r^{N+1} \mathrm{~d} r \\
& =-(N+1) \omega_{N-2} \int_{0}^{\infty} r^{N} F(w) \mathrm{d} r \\
& =-(N+1) \int_{R^{N-1}} F(w)\left|y^{\prime}\right|^{2} \mathrm{~d} y^{\prime} .
\end{aligned}
$$

Since $w$ satisfies

$$
\Delta w-w+f(w)=0, \quad y \in R^{N},
$$

by multiplying (2.17) by $\left|y^{\prime}\right|^{2}(\nabla w \cdot y)$ and integrating it with respect to $y^{\prime}$ in $R^{N-1}$ we obtain (2.12). 


$$
\begin{aligned}
\int_{R^{N-1}}\left|y^{\prime}\right|^{2} w \Delta w \mathrm{~d} y^{\prime} & =\omega_{N-2} \int_{0}^{\infty} w(r)\left(w^{\prime \prime}(r)+\frac{N-1}{r} w^{\prime}(r)\right) r^{N} \mathrm{~d} r \\
& =-\omega_{N-2} \int_{0}^{\infty} r^{N}\left(w^{\prime}(r)\right)^{2}+\frac{N-1}{2} \omega_{N-2} \int_{0}^{\infty} r^{N-2} w^{2} \mathrm{~d} r \\
& =-(N+1) \gamma_{1}+\frac{N-1}{2} \int_{R^{N-1}} w^{2} \mathrm{~d} y^{\prime} .
\end{aligned}
$$

Multiply (2.17) by $\left|y^{\prime}\right|^{2} w$ and integrate it in $R^{N-1}$. Then (2.13) is derived.

This proves Lemma 2.5.

LEMMA 2.6. - For any function $G(t)$ in $C^{1+\sigma}([0, \infty))$ with $G(0)=$ $G^{\prime}(0)=0$, we have

$$
\begin{aligned}
& \int_{\Omega_{\varepsilon, P}} G(w(y)) \mathrm{d} y \\
& \quad=\int_{R_{+}^{N}} G(w(y)) \mathrm{d} y-\varepsilon H(P) \frac{1}{2} \int_{R^{N-1}} G\left(w\left(y^{\prime}, 0\right)\right)\left|y^{\prime}\right|^{2} \mathrm{~d} y+\mathrm{o}(\varepsilon) .
\end{aligned}
$$

Proof. - Since $w$ decays exponentially in $y$ at infinity, we have

$$
\begin{aligned}
\int_{\Omega_{\varepsilon, P}} G(w(y)) \mathrm{d} y=\int_{\left(\Omega_{0}\right)_{\varepsilon, P}} G(w(y)) \mathrm{d} y+\mathrm{o}(\varepsilon) \\
=\int_{B^{+}\left(\frac{R_{0}}{\varepsilon}\right)} G(w(y)) \mathrm{d} y-\int_{B^{+}\left(\frac{R_{0}}{\varepsilon}\right) \backslash\left(\Omega_{0}\right)_{\varepsilon, P}} G(w(y)) \mathrm{d} y+\mathrm{o}(\varepsilon) \\
=\int_{R_{+}^{N}} G(w(y)) \mathrm{d} y-\int_{\left|y^{\prime}\right| \leqslant \frac{R_{0}}{\varepsilon}} \int_{0}^{\frac{1}{\varepsilon} \rho\left(\varepsilon y^{\prime}\right)} \int_{R_{+}^{N}} G\left(w\left(y^{\prime}, y_{N}\right)\right) \mathrm{d} y_{N} \mathrm{~d} y^{\prime}+\mathrm{o}(\varepsilon) \\
=\int_{R^{N} \rho\left(\varepsilon y^{\prime}\right)} G(w(y)) \mathrm{d} y-\int_{\left|y^{\prime}\right| \leqslant \frac{R_{0}}{\varepsilon}} G\left(w\left(y^{\prime}, 0\right)\right) \mathrm{d} y_{N} \mathrm{~d} y^{\prime} \\
\quad \int_{\left|y^{\prime}\right| \leqslant \frac{R_{0}}{\varepsilon}} \int_{0}^{\frac{1}{\varepsilon} \rho\left(\varepsilon y^{\prime}\right)}\left(G\left(w\left(y^{\prime}, y_{N}\right)-G\left(w\left(y^{\prime}, 0\right)\right)\right) \mathrm{d} y_{N} \mathrm{~d} y^{\prime}+\mathrm{o}(\varepsilon)\right.
\end{aligned}
$$




$$
\begin{aligned}
= & \int_{R_{+}^{N}} G(w(y)) \mathrm{d} y-\int_{\left|y^{\prime}\right| \leqslant \frac{R_{0}}{\varepsilon}} G\left(w\left(y^{\prime}, 0\right)\right) \frac{\rho\left(\varepsilon y^{\prime}\right)}{\varepsilon} \mathrm{d} y^{\prime} \\
& +\int_{\left|y^{\prime}\right| \leqslant \frac{R_{0}}{\varepsilon}} \mathrm{O}\left(\left|w\left(y^{\prime}, 0\right)\right|^{\sigma}\left(\frac{\rho\left(\varepsilon y^{\prime}\right)}{\varepsilon}\right)^{2}\right) \mathrm{d} y^{\prime}+\mathrm{o}(\varepsilon) \\
= & \int_{R_{+}^{N}} G(w(y)) \mathrm{d} y-\frac{1}{2} \varepsilon \int_{\left|y^{\prime}\right| \leqslant \frac{R_{0}}{\varepsilon}} G\left(w\left(y^{\prime}, 0\right)\right) \sum_{i, j=1}^{N-1} \rho_{i j}(0) y_{i} y_{j} \mathrm{~d} y^{\prime} \\
& +\int_{\left|y^{\prime}\right| \leqslant \frac{R_{0}}{\varepsilon}} \mathrm{O}\left(\varepsilon^{2}\left|y^{\prime}\right|^{3}\right) \mathrm{d} y^{\prime}+\mathrm{o}(\varepsilon) \\
= & \int_{R_{+}^{N}} G(w(y)) \mathrm{d} y-\varepsilon H(P) \frac{1}{2} \int_{\left|y^{\prime}\right| \leqslant \frac{R_{0}}{\varepsilon}} G\left(w\left(y^{\prime}, 0\right)\right)\left|y^{\prime}\right|^{2} \mathrm{~d} y^{\prime}+\mathrm{o}(\varepsilon) \\
= & \int_{R_{+}^{N}} G(w(y)) \mathrm{d} y-\varepsilon H(P) \frac{1}{2} \int_{R^{N-1}} G\left(w\left(y^{\prime}, 0\right)\right)\left|y^{\prime}\right|^{2} \mathrm{~d} y^{\prime} \\
& +\mathrm{o}(\varepsilon),
\end{aligned}
$$

where

$$
B^{+}\left(\frac{R_{0}}{\varepsilon}\right)=B\left(\frac{R_{0}}{\varepsilon}\right) \cap R_{+}^{N} \quad \text { and } \quad\left(\Omega_{0}\right)_{\varepsilon, P}=\left\{y \mid \varepsilon y+P \in \Omega_{0}\right\} .
$$

Hence Lemma 2.6 is proven.

LEMMA 2.7.-

$$
\int_{R_{+}^{N}} f(w)\left(P_{\Omega_{\varepsilon, P}} w-w\right)=\varepsilon H(P) \frac{N-1}{4} \int_{R^{N-1}}|w|^{2} \mathrm{~d} y^{\prime}+\mathrm{o}(\varepsilon) .
$$

Proof. - Using (2.5), (2.17) and the exponential decay of $w$ and $v_{1}$, we have

$$
\begin{aligned}
& \int_{R_{+}^{N}} f(w(y)) v_{1}(y) \mathrm{d} y=\int_{R_{+}^{N}}(w-\Delta w) v_{1}(y) \mathrm{d} y \\
& \quad=\int_{R_{+}^{N}}\left(w\left(v_{1}-\Delta v_{1}\right)\right)+\int_{R^{N-1}}\left(v_{1} \frac{\partial w}{\partial y_{N}}-w \frac{\partial v_{1}}{\partial y_{N}}\right) \mathrm{d} y^{\prime}
\end{aligned}
$$




$$
\begin{aligned}
& =\frac{1}{2} \int_{R^{N-1}} w(r) w^{\prime}(r) r^{-1} \sum_{i, j=1}^{N-1} \rho_{i j}(0) y_{i} y_{j} \mathrm{~d} y^{\prime} \\
& =\frac{1}{2} \int_{R^{N-1}} w(r) w^{\prime}(r) r^{-1} \sum_{i=1}^{N-1} \rho_{i i}(0)\left|y_{i}\right|^{2} \mathrm{~d} y^{\prime} \\
& =\frac{1}{2} H(P) \omega_{N-2} \int_{0}^{\infty} w(r) w^{\prime}(r) r^{N-1} \mathrm{~d} r \\
& =-\frac{N-1}{4} H(P) \int_{R^{N-1}} w^{2} \mathrm{~d} y^{\prime} .
\end{aligned}
$$

In view of Proposition 2.1, Lemma 2.7 follows immediately.

The next lemma is the key result in this section.

LEMMA 2.8. - For any $\mathbf{P}=\left(P_{1}, \ldots, P_{K}\right) \in \bar{\Lambda}$ and $\varepsilon$ sufficiently small

$$
\begin{aligned}
& J_{\varepsilon}\left(\sum_{i=1}^{K} P w_{i}\right) \\
&=\varepsilon^{N}\left[\frac{K}{2} I(w)-\varepsilon\left(\gamma_{1}+o(1)\right) \sum_{i=1}^{K} H\left(P_{i}\right)\right. \\
&\left.-\frac{1}{2} \sum_{k, l=1, k \neq l}^{K}\left(\gamma_{k l}+o(1)\right) w\left(\frac{\left|P_{k}-P_{l}\right|}{\varepsilon}\right)+o(\varepsilon)\right],
\end{aligned}
$$

where $\gamma_{1}$ is defined in (2.11) $\gamma_{k l}=\gamma_{l k} \in \Sigma$ and $\Sigma$ is defined by (2.8) and $\gamma_{1}$ is defined in (2.11).

Furthermore, if $w\left(\left|P_{k}-P_{l}\right| / \varepsilon\right)=\eta \varepsilon$, we have $\gamma_{k l} \in \Sigma_{1}$ where $\Sigma_{1}$ is defined by (2.9).

Proof. - We shall prove the case when $K=2$. The other cases are similar.

Since $\mathbf{P}=\left(P_{1}, P_{2}\right) \in \Lambda$, we have that $w\left(\left|P_{1}-P_{2}\right| / \varepsilon\right)<\eta \varepsilon$.

First we look at the case $K=1$. Note that by Proposition 2.1, Lemmas 2.6 and 2.7 we have

$$
\begin{aligned}
& \varepsilon^{2} \int_{\Omega} \mid \nabla\left(\left.P_{\Omega_{\varepsilon, P}} w\left(\frac{x-P}{\varepsilon}\right)\right|^{2}+\int_{\Omega}\left|P_{\Omega_{\varepsilon, P}} w\left(\frac{x-P}{\varepsilon}\right)\right|^{2}\right. \\
& \quad=\varepsilon^{N} \int_{\Omega_{\varepsilon, P}} f(w) P_{\Omega_{\varepsilon, P}} w
\end{aligned}
$$


C. GUI ET AL. / Ann. Inst. Henri Poincaré 17 (2000) 47-82

$$
\begin{aligned}
= & \varepsilon^{N}\left(\int_{\Omega_{\varepsilon, P}} f(w) w+\int_{\Omega_{\varepsilon, P}} f(w)\left(P_{\Omega_{\varepsilon, P}} w-w\right)\right) \\
= & \varepsilon^{N}\left(\int_{R_{+}^{N}} f(w) w-\varepsilon H(P) \frac{1}{2} \int_{R^{N-1}} f(w) w\left|y^{\prime}\right|^{2} \mathrm{~d} y^{\prime}\right. \\
& \left.+\varepsilon H(P) \frac{N-1}{4} \int_{R^{N-1}}|w|^{2} \mathrm{~d} y^{\prime}+\mathrm{o}(\varepsilon)\right) .
\end{aligned}
$$

Similarly we have

$$
\begin{aligned}
\int_{\Omega} & F\left(P_{\Omega_{\varepsilon, P}} w\left(\frac{x-P}{\varepsilon}\right)\right) \mathrm{d} x \\
= & \varepsilon^{N}\left(\int_{\Omega_{\varepsilon, P}} F(w) \mathrm{d} y+\int_{\Omega_{\varepsilon, P}}\left(F\left(P_{\Omega_{\varepsilon, P}} w\right)-F(w)\right) \mathrm{d} y\right) \\
= & \varepsilon^{N}\left(\int_{R_{+}^{N}} F(w) \mathrm{d} y-\varepsilon H(P) \frac{1}{2} \int_{R^{N-1}} f(w) w\left|y^{\prime}\right|^{2} \mathrm{~d} y^{\prime}\right. \\
& \left.+\varepsilon H(P) \frac{N-1}{4} \int_{R^{N-1}}|w|^{2} \mathrm{~d} y^{\prime}+\mathrm{o}(\varepsilon)\right) .
\end{aligned}
$$

Then

$$
J_{\varepsilon}\left(P_{\Omega_{\varepsilon, P}} w\right)=\varepsilon^{N}\left(\frac{1}{2} I(w)-\gamma_{1} H(P)+o(\varepsilon)\right) .
$$

For the case $K=2$, we can write

$$
\begin{aligned}
\int_{\Omega} F\left(P w_{1}+P w_{2}\right)= & \int_{\Omega_{1}} F\left(P w_{1}+P w_{2}\right)+\int_{\Omega_{2}} F\left(P w_{1}+P w_{2}\right) \\
& +\int_{\Omega_{3}} F\left(P w_{1}+P w_{2}\right) \\
= & I_{1}+I_{2}+I_{3},
\end{aligned}
$$

where $I_{i}, i=1,2,3$, are defined at the last equality and

$$
\begin{gathered}
\Omega_{1}=\left\{\left|x-P_{1}\right| \leqslant \frac{1}{2}\left|P_{1}-P_{2}\right|\right\}, \quad \Omega_{2}=\left\{\left|x-P_{2}\right| \leqslant \frac{1}{2}\left|P_{1}-P_{2}\right|\right\}, \\
\Omega_{3}=\Omega \backslash\left(\Omega_{1} \cup \Omega_{2}\right) .
\end{gathered}
$$

For $I_{3}$, we have 


$$
\begin{aligned}
\left|\varepsilon^{-N} I_{3}\right| & \leqslant C \int_{\left(\Omega_{3}\right)_{\varepsilon}}\left(w_{1}+w_{2}\right)^{2+\sigma}=\mathrm{O}\left(w\left(\frac{\left|P_{1}-P_{2}\right|}{\varepsilon}\right)^{1+0.5 \sigma}\right) \\
& =\mathrm{O}\left(\varepsilon^{1+0.5 \sigma}\right) .
\end{aligned}
$$

For $I_{1}$, using $w\left(\left|P_{1}-P_{2}\right| / \varepsilon\right)=\mathrm{O}(\varepsilon)$ we have

$$
\begin{aligned}
\varepsilon^{-N} I_{1}= & \int_{\left(\Omega_{1}\right)_{\varepsilon}}\left(F\left(P w_{1}\right)+f\left(P w_{1}\right) P w_{2}\right)+\mathrm{O}\left(\varepsilon^{1+0.5 \sigma}\right) \\
= & \int_{\Omega_{\varepsilon}} F\left(P w_{1}\right)+\int_{\left(\Omega_{1}\right)_{\varepsilon}} f\left(w_{1}\right) w_{2}+\mathrm{O}\left(\varepsilon^{1+0.5 \sigma}\right) \\
= & \int_{R_{+}^{N}} F(w)-\varepsilon H\left(P_{1}\right)\left(\frac{1}{2} \int_{R^{N-1}} F(w)\left|y^{\prime}\right|^{2} \mathrm{~d} y^{\prime}\right. \\
& \left.-\frac{N-1}{4} \int_{R^{N-1}}|w|^{2} \mathrm{~d} y^{\prime}\right)+\int_{\left(\Omega_{1}\right)_{\varepsilon}} f\left(w_{1}\right) w_{2}+\mathrm{O}\left(\varepsilon^{1+0.5 \sigma}\right) .
\end{aligned}
$$

Similarly,

$$
\begin{aligned}
\varepsilon^{-N} I_{2}= & \int_{R_{+}^{N}} F(w)-\varepsilon H\left(P_{2}\right)\left(\frac{1}{2} \int_{R^{N-1}} F(w)\left|y^{\prime}\right|^{2} \mathrm{~d} y^{\prime}\right. \\
& \left.-\frac{N-1}{4} \int_{R^{N-1}}|w|^{2} \mathrm{~d} y^{\prime}\right)+\int_{\left(\Omega_{2}\right)_{\varepsilon}} f\left(w_{2}\right) w_{1}+\mathrm{O}\left(\varepsilon^{1+0.5 \sigma}\right) .
\end{aligned}
$$

\section{Hence}

$$
\begin{aligned}
\varepsilon^{-N} J_{\varepsilon}\left(\sum_{i=1}^{K} P w_{i}\right) \\
=\int_{\Omega_{\varepsilon}}\left[\frac{1}{2}\left(\sum_{i=1}^{2}\left(\left|\nabla P w_{i}\right|^{2}+\left(P w_{i}\right)^{2}\right)\right)+\nabla P w_{1} \nabla P w_{2}+P w_{1} P w_{2}\right] \\
\quad-\int_{\Omega_{\varepsilon}} F\left(P w_{1}+P w_{2}\right) \\
=\int_{\Omega_{\varepsilon}}\left[\frac{1}{2}\left(\sum_{i=1}^{2}\left(\left|\nabla P w_{i}\right|^{2}+\left(P w_{i}\right)^{2}\right)\right)\right]+\int_{\Omega_{\varepsilon}} f\left(w_{1}\right) P w_{2}
\end{aligned}
$$




$$
\begin{aligned}
& -\int_{\Omega_{\varepsilon}} F\left(P w_{1}+P w_{2}\right) \\
= & 2 I(w)-\gamma_{1} \varepsilon \sum_{i=1}^{2} H\left(P_{i}\right)+\int_{\Omega_{\varepsilon}} f\left(w_{1}\right) P w_{2}-\int_{\left(\Omega_{1}\right)_{\varepsilon}} f\left(w_{1}\right) w_{2} \\
& -\int_{\left(\Omega_{2}\right)_{\varepsilon}} f\left(w_{2}\right) w_{1}+\mathrm{o}\left(w\left(\frac{\left|P_{1}-P_{2}\right|}{\varepsilon}\right)+\mathrm{O}\left(\varepsilon^{1+0.5 \sigma}\right)\right) \\
= & 2 I(w)-\gamma_{1} \varepsilon \sum_{i=1}^{2} H\left(P_{i}\right)-\left(\gamma_{12}+\mathrm{o}(1)\right) w\left(\frac{\left|P_{1}-P_{2}\right|}{\varepsilon}\right) \\
& +\mathrm{O}\left(\varepsilon^{1+0.5 \sigma}\right) .
\end{aligned}
$$

Here we have used Lemma 2.4

$$
\int_{\Omega_{\varepsilon}} f\left(w_{1}\right) w_{2}=\left(\gamma_{12}+\mathrm{o}(1)\right) w\left(\frac{\left|P_{1}-P_{2}\right|}{\varepsilon}\right)
$$

and similarly

$$
\begin{aligned}
\int_{\left(\Omega_{1}\right)_{\varepsilon}} f\left(w_{1}\right) w_{2} & =\left(\gamma_{12}+o(1)\right) w\left(\frac{\left|P_{1}-P_{2}\right|}{\varepsilon}\right), \\
\int_{\left(\Omega_{2}\right)_{\varepsilon}} f\left(w_{2}\right) w_{1} & =\left(\gamma_{21}+o(1)\right) w\left(\frac{\left|P_{1}-P_{2}\right|}{\varepsilon}\right) \\
& =\left(\gamma_{12}+o(1)\right) w\left(\frac{\left|P_{1}-P_{2}\right|}{\varepsilon}\right) .
\end{aligned}
$$

\section{LIAPUNOV-SCHMIDT REDUCTION}

In this section, we reduce problem (1.2) to finite dimensions by the Liapunov-Schmidt method. We first introduce some notations.

Let $H_{N}^{2}\left(\Omega_{\varepsilon}\right)$ be the Hilbert space defined by

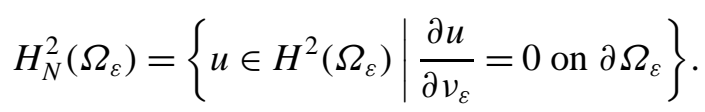


Define

$$
S_{\varepsilon}(u)=\Delta u-u+f(u)
$$

for $u \in H_{N}^{2}\left(\Omega_{\varepsilon}\right)$. Then solving Eq. (1.2) is equivalent to

$$
S_{\varepsilon}(u)=0, \quad u \in H_{N}^{2}\left(\Omega_{\varepsilon}\right) \text {. }
$$

Fix $\mathbf{P}=\left(P_{1}, \ldots, P_{K}\right) \in \bar{\Lambda}$. To study (1.2) we first consider the linearized operator

$$
\begin{gathered}
\tilde{L_{\varepsilon}}: u \mapsto \Delta u-u+f^{\prime}\left(\sum_{i=1}^{K} P w_{i}\right) u, \\
H_{N}^{2}\left(\Omega_{\varepsilon}\right) \rightarrow L^{2}\left(\Omega_{\varepsilon}\right) .
\end{gathered}
$$

It is easy to see (integration by parts) that the cokernel of ${\tilde{L_{\varepsilon}}}_{\varepsilon}$ coincides with its kernel. Choose approximate cokernel and kernel as

$$
\begin{aligned}
\mathcal{C}_{\varepsilon, \mathbf{P}} & =\mathcal{K}_{\varepsilon, \mathbf{P}} \\
& =\operatorname{span}\left\{\frac{\partial P w_{i}}{\partial \tau_{P_{i, j}}} \mid i=1, \ldots, K, j=1, \ldots, N-1\right\} .
\end{aligned}
$$

Let $\pi_{\varepsilon, \mathbf{P}}$ denote the projection from $L^{2}\left(\Omega_{\varepsilon}\right)$ onto $\mathcal{C}_{\varepsilon, \mathbf{P}}^{\perp}$. Our goal in this section is to show that the equation

$$
\pi_{\varepsilon, \mathbf{P}} \circ S_{\varepsilon}\left(\sum_{i=1}^{K} P w_{i}+\Phi_{\varepsilon, \mathbf{P}}\right)=0
$$

has a unique solution $\Phi_{\varepsilon, \mathbf{P}} \in \mathcal{K}_{\varepsilon, \mathbf{P}}^{\perp}$ if $\varepsilon$ is small enough and $\mathbf{P}=$ $\left(P_{1}, \ldots, P_{K}\right) \in \bar{\Lambda}$.

As a preparation in the following two propositions we show the invertibility of the corresponding linearized operator.

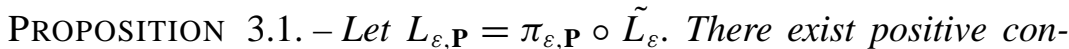
stants $\bar{\varepsilon}, \bar{\lambda}$ such that for all $\varepsilon \in(0, \bar{\varepsilon})$ and $\mathbf{P}=\left(P_{1}, \ldots, P_{K}\right) \in \bar{\Lambda}$

$$
\left\|L_{\varepsilon, \mathbf{P}} \Phi\right\|_{L^{2}\left(\Omega_{\varepsilon}\right)} \geqslant \lambda\|\Phi\|_{H^{2}\left(\Omega_{\varepsilon}\right)}
$$

for all $\Phi \in \mathcal{K}_{\varepsilon, \mathbf{P}}^{\perp}$

Proposition 3.2. - For any $\varepsilon \in(0, \tilde{\varepsilon})$ and $\mathbf{P}=\left(P_{1}, \ldots, P_{K}\right) \in \bar{\Lambda}$ the map

$$
L_{\varepsilon, \mathbf{P}}=\pi_{\varepsilon, \mathbf{P}} \circ \tilde{L}_{\varepsilon}: \mathcal{K}_{\varepsilon, \mathbf{P}}^{\perp} \rightarrow \mathcal{C}_{\varepsilon, \mathbf{P}}^{\perp}
$$


is surjective.

Proof of Proposition 3.1. - We will follow the method used in [7,25, 26], and [37]. Suppose that (3.1) is false. Then there exist sequences $\left\{\varepsilon_{k}\right\},\left\{\mathbf{P}_{\mathbf{k}}\right\}=\left\{\left(P_{1, k}, \ldots, P_{K, k}\right)\right\}$, and $\left\{\Phi_{k}\right\}(i=1,2, \ldots, K, k=1,2, \ldots)$ with $\varepsilon_{k}>0, \mathbf{P}_{\mathbf{k}} \in \bar{\Lambda}, \Phi_{k} \in \mathcal{K}_{\varepsilon_{k}}^{\perp} \mathbf{P}_{\mathbf{k}}$ such that

$$
\begin{aligned}
& \varepsilon_{k} \rightarrow 0, \\
& \mathbf{P}_{\mathbf{k}} \rightarrow \mathbf{P} \in \bar{\Lambda}, \\
& \left\|L_{\varepsilon_{k}, \mathbf{P}_{\mathbf{k}}} \Phi_{k}\right\|_{L^{2}\left(\Omega_{\varepsilon_{k}}\right)} \rightarrow 0, \\
& \left\|\Phi_{k}\right\|_{H^{2}\left(\Omega_{\varepsilon_{k}}\right)}=1, \quad k=1,2, \ldots
\end{aligned}
$$

For $j=1,2, \ldots, N-1$ denote

$$
e_{i j, k}=\frac{\partial}{\partial \tau_{\left(P_{i, k}\right)_{j}}} P w_{i, k} /\left\|\frac{\partial}{\partial \tau_{\left(P_{i, k}\right)_{j}}} P w_{i, k}\right\|_{L^{2}\left(\Omega_{\varepsilon_{k}}\right)},
$$

where

$$
P w_{i, k}(y)=P_{\Omega_{\varepsilon_{k}, P_{i, k}}} w\left(y-\frac{P_{i, k}}{\varepsilon_{k}}\right), \quad y \in \Omega_{\varepsilon_{k}} .
$$

Note that

$$
\left\langle e_{i_{1} j_{1}, k}, e_{i_{2} j_{2}, k}\right\rangle=\delta_{i_{1} i_{2}} \delta_{j_{1} j_{2}}+\mathcal{O}\left(\varepsilon_{k}\right) \quad \text { as } k \rightarrow \infty
$$

by Proposition 2.2, the symmetry of the function $w$ and the fact that $\mathbf{P} \in \bar{\Lambda}$ (recall that $\left.w\left(\left|P_{k}-P_{l}\right| / \varepsilon\right) \leqslant \eta \varepsilon\right)$. Here $\delta_{i_{1} i_{2}}$ is the Kronecker symbol. Furthermore, because of (3.4),

$$
\left\|\tilde{L}_{\varepsilon_{k}} \Phi_{k}\right\|_{L^{2}}^{2}-\sum_{i=1}^{K} \sum_{j=1}^{N-1}\left(\int_{\Omega_{\varepsilon_{k}}} \tilde{L}_{\varepsilon_{k}} \Phi_{k} e_{i j, k}\right)^{2} \rightarrow 0
$$

as $k \rightarrow \infty$. Let $\Omega_{0}, \chi, \rho$ and $T$ be as defined in Section 2. (Note that we allow $R_{0} \rightarrow 0$ but $R_{0} / \varepsilon \rightarrow \infty$.) Then $T$ has an inverse $T^{-1}$ such that

$$
T^{-1}: T\left(B\left(P, R_{0}\right) \cap \bar{\Omega}\right) \rightarrow B\left(P, R_{0}\right) \cap \bar{\Omega} .
$$

Recall that $\varepsilon y=T(x)$. We use the notation $T^{(i)}$ if $P$ is replaced by $P_{i}$. We introduce new sequences $\left\{\varphi_{i, k}\right\}$ by

$$
\varphi_{i, k}(y)=\chi\left(\frac{1}{\varepsilon_{k}}\left(T^{(i)}\right)^{-1}\left(\varepsilon_{k} y\right)\right) \Phi_{k}\left(\left(T^{(i)}\right)^{-1}\left(\varepsilon_{k} y\right)\right)
$$


for $y \in R_{+}^{N}$. Since $T^{(i)}$ and $\left(T^{(i)}\right)^{-1}$ have bounded derivatives it follows from (3.5) and the smoothness of $\chi$ that

$$
\left\|\varphi_{i, k}\right\|_{H^{2}\left(R_{+}^{N}\right)} \leqslant C
$$

for all $k$ sufficiently large. Since also

$$
\left\|\varphi_{i, k}\right\|_{H^{2}\left(R_{+}^{N} \backslash B(0, R)\right)} \rightarrow 0 \quad \text { as } R \rightarrow \infty
$$

uniformly in $k$ for all $k$ large enough there exists a subsequence, again denoted by $\left\{\varphi_{i, k}\right\}$ which converges weakly in $H^{2}\left(R_{+}^{N}\right)$ to a limit $\varphi_{i, \infty}$ as $k \rightarrow \infty$. We are now going to show that $\varphi_{i, \infty} \equiv 0$. As a first step we deduce

$$
\int_{R_{+}^{N}} \varphi_{i, \infty} \frac{\partial w}{\partial y_{j}}=0, \quad j=1, \ldots, N-1 .
$$

This statement is shown as follows (note that $\operatorname{det} D T=\operatorname{det} D T^{-1}=1$ )

$$
\begin{aligned}
\int_{R_{+}^{N}} \varphi_{i, k}(y)\left[\frac{\partial P w_{i, k}}{\partial \tau_{\left(P_{i, k}\right)_{j}}}\left(\frac{\left(T^{(i)}\right)^{-1}\left(\varepsilon_{k} y\right)}{\varepsilon_{k}}\right)\right] \mathrm{d} y \\
=\varepsilon_{k}^{-N} \int_{\Omega_{0}} \chi\left(x-P_{i, k}\right) \Phi_{k}\left(\frac{x}{\varepsilon_{k}}\right) \frac{\partial P w_{i, k}}{\partial \tau_{\left(P_{i, k}\right)_{j}}}\left(\frac{x-P_{i, k}}{\varepsilon_{k}}\right) \mathrm{d} x \\
=\varepsilon_{k}^{-N} \int_{\Omega} \Phi_{k}\left(\frac{x}{\varepsilon_{k}}\right) \frac{\partial P w_{i, k}}{\partial \tau_{\left(P_{i, k}\right)_{j}}}\left(\frac{x-P_{i, k}}{\varepsilon_{k}}\right) \\
-\varepsilon_{k}^{-N} \int_{\Omega \backslash \Omega_{0}} \Phi_{k}\left(\frac{x}{\varepsilon_{k}}\right) \frac{\partial P w_{i, k}}{\partial \tau_{\left(P_{i, k}\right)_{j}}}\left(\frac{x-P_{i, k}}{\varepsilon_{k}}\right) \\
\quad-\varepsilon_{k}^{-N}\left[1-\chi\left(x-P_{i, k}\right)\right] \Phi_{k}\left(\frac{x}{\varepsilon_{k}}\right) \frac{\partial P w_{i, k}}{\partial \tau_{\left(P_{i, k}\right)_{j}}}\left(\frac{x-P_{i, k}}{\varepsilon_{k}}\right) \\
=0-\varepsilon_{k}^{-N} \int_{\Omega \backslash \Omega_{0}} \Phi_{k}\left(\frac{x}{\varepsilon_{k}}\right)\left[\frac{\partial w}{\partial\left(P_{i, k}\right)_{j}}\left(\frac{x-P_{i, k}}{\varepsilon_{k}}\right)\right. \\
\left.\quad-\frac{\partial P_{w_{i, k}}}{\partial \tau_{\left(P_{i, k}\right)_{j}}}\left(\frac{x-P_{i, k}}{\varepsilon_{k}}\right)\right]-\varepsilon_{k}^{-N} \int_{\Omega_{0}}\left[1-\chi\left(x-P_{i, k}\right)\right] \Phi_{k}\left(\frac{x}{\varepsilon_{k}}\right) \\
\quad \times\left[\frac{\partial w}{\partial\left(P_{i, k}\right)_{j}}\left(\frac{x-P_{i, k}}{\varepsilon_{k}}\right)-\frac{\partial P w_{i, k}}{\partial\left(P_{i, k}\right)_{j}}\left(\frac{x-P_{i, k}}{\varepsilon_{k}}\right)\right]
\end{aligned}
$$




$$
\begin{aligned}
& -\varepsilon_{k}^{-N} \int_{\Omega \backslash \Omega_{0}} \Phi_{k}\left(\frac{x}{\varepsilon_{k}}\right) \frac{\partial w}{\partial\left(P_{i, k}\right)_{j}}\left(\frac{x-P_{i, k}}{\varepsilon_{k}}\right) \\
& -\varepsilon_{k}^{-N} \int_{\Omega_{0}}\left[1-\chi\left(x-P_{i, k}\right)\right] \Phi_{k}\left(\frac{x}{\varepsilon_{k}}\right) \frac{\partial w}{\partial\left(P_{i, k}\right)_{j}}\left(\frac{x-P_{i, k}}{\varepsilon_{k}}\right),
\end{aligned}
$$

where $\Omega_{0}$ is as defined in Section 2. In the last expression the first two terms tend to zero as $k \rightarrow \infty$ since $\varepsilon_{k}{ }^{-N} \Phi_{k}$ is bounded in $L^{2}(\Omega)$ and the term in the square bracket converges to 0 strongly in $L^{2}(\Omega)$. The last two terms tend to zero as $k \rightarrow \infty$ because of the exponential decay of $\partial w / \partial\left(P_{i, k}\right)_{j}$ at infinity.

We conclude

$$
\begin{aligned}
& \underset{k \rightarrow \infty}{\limsup }\left|\int_{R_{+}^{N}} \varphi_{i, k}(y)\left[\frac{\partial P w_{i, k}}{\partial \tau_{\left(P_{i, k}\right)_{j}}}\left(\frac{\left(T^{(i)}\right)^{-1}\left(\varepsilon_{k} y\right)}{\varepsilon_{k}}\right)\right]\right|=0, \\
& i=1, \ldots, K, j=1, \ldots, N-1 .
\end{aligned}
$$

This implies (3.8).

Let $\mathcal{K}_{0}$ and $\mathcal{C}_{0}$ be the kernel and cokernel, respectively, of the linear operator $S_{0}^{\prime}(w)$ which is the Fréchet derivative at $w$ of

$$
\begin{aligned}
& S_{0}(v)=\Delta v-v+f(v), \\
& S_{0}: H_{N}^{2}\left(R_{+}^{N}\right) \rightarrow L^{2}\left(R_{+}^{N}\right),
\end{aligned}
$$

where

$$
H_{N}^{2}\left(R_{+}^{N}\right)=\left\{u \in H_{N}^{2}\left(R_{+}^{N}\right) \mid \frac{\partial u}{\partial y_{N}}=0\right\} .
$$

Note that

$$
\begin{gathered}
S_{0}^{\prime}(w) v=\Delta v-v+f^{\prime}(w) v, \\
\mathcal{K}_{0}=\mathcal{C}_{0}=\operatorname{span}\left\{\frac{\partial w}{\partial y_{j}} \mid j=1, \ldots, N-1\right\} .
\end{gathered}
$$

Eq. (3.8) implies that $\varphi_{i, \infty} \in \mathcal{K}_{0}^{\perp}$. By the exponential decay of $w$ and by (3.4) we have after possibly taking a further subsequence that

$$
\Delta \varphi_{i, \infty}-\varphi_{i, \infty}+f^{\prime}(w) \varphi_{i, \infty}=0
$$

i.e., $\varphi_{i, \infty} \in \mathcal{K}_{0}$. Therefore $\varphi_{i, \infty}=0$.

Hence

$$
\varphi_{i, k} \rightarrow 0 \quad \text { weakly in } H^{2}\left(R_{+}^{N}\right) \text { as } k \rightarrow \infty \text {. }
$$


By the definition of $\varphi_{i, k}$ we get $\Phi_{k} \rightarrow 0$ in $H^{2}$ and

$$
\left\|\Phi_{k}\right\|_{L^{2}\left(\Omega_{\varepsilon_{k}}\right)} \rightarrow 0 \quad \text { as } k \rightarrow \infty .
$$

Furthermore,

$$
\left\|f^{\prime}\left(\sum_{i=1}^{K} P w_{i}\right) \Phi_{k}\right\|_{L^{2}\left(\Omega_{\varepsilon_{k}}\right)} \rightarrow 0
$$

and therefore

$$
\left\|(\Delta-1) \Phi_{k}\right\|_{L^{2}\left(\Omega_{\varepsilon_{k}}\right)} \rightarrow 0 \quad \text { as } k \rightarrow \infty .
$$

Since

$$
\int_{\Omega_{\varepsilon_{k}}}\left|\nabla \Phi_{k}\right|^{2}+\Phi_{k}^{2}=\int_{\Omega_{\varepsilon_{k}}}\left[(1-\Delta) \Phi_{k}\right] \Phi_{k} \leqslant C\left\|(\Delta-1) \Phi_{k}\right\|_{L^{2}\left(\Omega_{\varepsilon_{k}}\right)}
$$

we have that

$$
\left\|\Phi_{k}\right\|_{H^{1}\left(\Omega_{\varepsilon_{k}}\right)} \rightarrow 0 \quad \text { as } k \rightarrow \infty .
$$

In summary:

$$
\left\|\Delta \Phi_{k}\right\|_{L^{2}\left(\Omega_{\varepsilon_{k}}\right)} \rightarrow 0 \quad \text { and } \quad\left\|\Phi_{k}\right\|_{H^{1}\left(\Omega_{\varepsilon_{k}}\right)} \rightarrow 0 .
$$

From (3.11) and the following elliptic regularity estimate (for a proof see Appendix B in [37])

$$
\left\|\Phi_{k}\right\|_{H^{2}\left(\Omega_{\varepsilon_{k}}\right)} \leqslant C\left(\left\|\Delta \Phi_{k}\right\|_{L^{2}\left(\Omega_{\varepsilon_{k}}\right)}+\left\|\Phi_{k}\right\|_{H^{1}\left(\Omega_{\varepsilon_{k}}\right)}\right)
$$

for $\Phi_{k} \in H_{N}^{2}\left(\Omega_{\varepsilon_{k}}\right)$ we deduce that

$$
\left\|\Phi_{k}\right\|_{H^{2}\left(\Omega_{\varepsilon_{k}}\right)} \rightarrow 0 \quad \text { as } k \rightarrow \infty .
$$

This contradicts the assumption

$$
\left\|\Phi_{k}\right\|_{H^{2}\left(\Omega_{\varepsilon_{k}}\right)}=1
$$

and the proof of Proposition 3.1 is completed.

Proof of Proposition 3.2. - We define a linear operator $T$ from $L^{2}\left(\Omega_{\varepsilon}\right)$ to itself by

$$
T=\pi_{\varepsilon, \mathbf{P}} \circ \tilde{L} \circ \pi_{\varepsilon, \mathbf{P}}
$$


Its domain of definition is $H_{N}^{2}\left(\Omega_{\varepsilon}\right)$. By the theory of elliptic equations and by integration by parts it is easy to see that $T$ is a (unbounded) selfadjoint operator on $L^{2}\left(\Omega_{\varepsilon}\right)$ and a closed operator. The $L^{2}$ estimates of elliptic equations imply that the range of $T$ is closed in $L^{2}\left(\Omega_{\varepsilon}\right)$. Then by the Closed Range Theorem ([39], p. 205), we know that the range of $T$ is the orthogonal complement of its kernal which is, by Proposition 3.1, $\mathcal{K}_{\varepsilon, \mathbf{P}}$. This leads to Proposition 3.2.

We are now in a position to solve the equation

$$
\pi_{\varepsilon, P} \circ S_{\varepsilon}\left(\sum_{i=1}^{K} P w_{i}+\Phi_{\varepsilon, \mathbf{P}}\right)=0 .
$$

Since $\left.L_{\varepsilon, \mathbf{P}}\right|_{\mathcal{K}_{\varepsilon, \mathbf{P}}^{\perp}}$ is invertible (call the inverse $L_{\varepsilon, \mathbf{P}}^{-1}$ ) we can rewrite

$$
\begin{aligned}
\Phi & =-\left(L_{\varepsilon, \mathbf{P}}^{-1} \circ \pi_{\varepsilon, \mathbf{P}}\right)\left(S_{\varepsilon}\left(\sum_{i=1}^{K} P w_{i}\right)\right)-\left(L_{\varepsilon, \mathbf{P}}^{-1} \circ \pi_{\varepsilon, \mathbf{P}}\right) N_{\varepsilon, \mathbf{P}}(\Phi) \\
& \equiv G_{\varepsilon, \mathbf{P}}(\Phi)
\end{aligned}
$$

where

$$
N_{\varepsilon, \mathbf{P}}(\Phi)=S_{\varepsilon}\left(\sum_{i=1}^{K} P w_{i}+\Phi\right)-\left[S_{\varepsilon}\left(\sum_{i=1}^{K} P w_{i}\right)+S_{\varepsilon}^{\prime}\left(\sum_{i=1}^{K} P w_{i}\right) \Phi\right]
$$

and the operator $G_{\varepsilon, \mathbf{P}}$ is defined by the last equation for $\Phi \in H_{N}^{2}\left(\Omega_{\varepsilon}\right)$. We are going to show that the operator $G_{\varepsilon, \mathbf{P}}$ is a contraction on

$$
B_{\varepsilon, \delta} \equiv\left\{\Phi \in H^{2}\left(\Omega_{\varepsilon}\right) \mid\|\Phi\|_{H^{2}\left(\Omega_{\varepsilon}\right)}<\delta\right\}
$$

if $\delta$ is small enough.

In fact we have the following lemma

LEMMA 3.3. - For $\varepsilon$ sufficiently small, we have

$$
\begin{aligned}
& \left|N_{\varepsilon, \mathbf{P}}\right| \leqslant C\left(\left|\Phi_{\varepsilon, \mathbf{P}}\right|^{1+\sigma}+\left|\Phi_{\varepsilon, \mathbf{P}}\right|^{p_{1}}\right), \\
& \left\|S_{\varepsilon}\left(\sum_{i=1}^{K} P w_{i}\right)\right\|_{L^{2}\left(\Omega_{\varepsilon}\right)} \leqslant C \varepsilon^{\frac{1+\sigma}{2}} .
\end{aligned}
$$

Proof. - (3.16) follows from the mean value theorem.

To prove (3.17), we divide the domain into $(K+1)$ parts: let $\Omega=$ $\bigcup_{i=1}^{K+1} \Omega_{i}$ where 


$$
\begin{aligned}
& \Omega_{i}=\left\{\left|x-P_{i}\right| \leqslant \frac{1-\delta}{2} \min _{k \neq l}\left|P_{k}-P_{l}\right|\right\}, \quad i=1, \ldots, K, \\
& \Omega_{K+1}=\Omega \backslash \bigcup_{i=1}^{K} \Omega_{i} .
\end{aligned}
$$

Note that

$$
S_{\varepsilon}\left(\sum_{i=1}^{K} P w_{i}\right)=f\left(\sum_{i=1}^{K} P w_{i}\right)-\sum_{i=1}^{K} f\left(w_{i}\right) .
$$

We now estimate $S_{\varepsilon}\left(\sum_{i=1}^{K} P w_{i}\right)$ in each domain. In $\Omega_{K+1}$, we have

$$
\left|S_{\varepsilon}\left(\sum_{i=1}^{K} P w_{i}\right)\right| \leqslant\left(w_{1}+\cdots+w_{K}\right)^{1+\sigma} \leqslant \mathrm{O}\left(\varepsilon^{\frac{1+\sigma}{2}}\right) .
$$

Hence, using also the fact that $w(y)$ decays exponentially in $|y|$ we obtain

$$
\left\|S_{\varepsilon}\left(\sum_{i=1}^{K} P w_{i}\right)\right\|_{L^{2}\left(\left(\Omega_{K+1}\right)_{\varepsilon}\right)} \leqslant \mathrm{O}\left(\varepsilon^{\frac{1+\sigma}{2}}\right)
$$

In $\Omega_{i}, i=1, \ldots, K$, we have

$$
\begin{aligned}
\left|S_{\varepsilon}\left(\sum_{i=1}^{K} P w_{i}\right)\right| \leqslant & \sum_{j \neq i}\left(\left|f^{\prime}\left(w_{i}\right) w_{j}\right|+\left|f^{\prime}\left(w_{i}\right)\left(P w_{j}-w_{j}\right)\right|\right) \\
& +\mathrm{O}\left(\sum_{j \neq i}\left(\left|P w_{j}\right|^{1+\sigma}+\left|w_{j}\right|^{1+\sigma}\right)\right) \\
& +\mathrm{O}\left(\left|P w_{i}-w_{i}\right|^{1+\sigma}\right) .
\end{aligned}
$$

Using Proposition 2.1 and the facts that $P w, w$ and $v_{1}$ decay exponentially, we obtain

$$
\left\|S_{\varepsilon}\left(\sum_{i=1}^{K} P w_{i}\right)\right\|_{L^{2}\left(\left(\Omega_{i}\right)_{\varepsilon}\right)} \leqslant \mathrm{O}\left(\varepsilon^{\frac{1+\sigma}{2}}\right) .
$$

Thus

$\left\|G_{\varepsilon, \mathbf{P}}(\Phi)\right\|_{H^{2}\left(\Omega_{\varepsilon}\right)}$ 


$$
\begin{aligned}
& \leqslant \lambda^{-1}\left(\left\|\pi_{\varepsilon, \mathbf{P}} \circ N_{\varepsilon, \mathbf{P}}(\Phi)\right\|_{L^{2}\left(\Omega_{\varepsilon}\right)}+\left\|\pi_{\varepsilon, \mathbf{P}} \circ\left(S_{\varepsilon}\left(\sum_{i=1}^{K} P w_{i}\right)\right)\right\|_{L^{2}\left(\Omega_{\varepsilon}\right)}\right) \\
& \leqslant \lambda^{-1} C\left(c(\delta) \delta+\varepsilon^{\frac{1+\sigma}{2}}\right),
\end{aligned}
$$

where $\lambda>0$ is independent of $\delta>0$ and $c(\delta) \rightarrow 0$ as $\delta \rightarrow 0$. Similarly we show

$$
\left\|G_{\varepsilon, \mathbf{P}}(\Phi)-G_{\varepsilon, \mathbf{P}}\left(\Phi^{\prime}\right)\right\|_{H^{2}\left(\Omega_{\varepsilon}\right)} \leqslant \lambda^{-1} C c(\delta)\left\|\Phi-\Phi^{\prime}\right\|_{H^{2}\left(\Omega_{\varepsilon}\right)},
$$

where $c(\delta) \rightarrow 0$ as $\delta \rightarrow 0$. Therefore $M_{\varepsilon, \mathbf{P}}$ is a contraction on $B_{\delta}$. The existence of a fixed point $\Phi_{\varepsilon, \mathbf{P}}$ now follows from the Contraction Mapping Principle and $\Phi_{\varepsilon, \mathbf{P}}$ is a solution of (3.14).

Because of

$$
\begin{aligned}
\left\|\Phi_{\varepsilon, \mathbf{P}}\right\|_{H^{2}(\Omega)} & \leqslant \lambda^{-1}\left(\left\|N_{\varepsilon, \mathbf{P}}\left(\Phi_{\varepsilon, \mathbf{P}}\right)\right\|_{L^{2}\left(\Omega_{\varepsilon}\right)}+\left\|S_{\varepsilon}\left(\sum_{i=1}^{K} P w_{i}\right)\right\|_{L^{2}\left(\Omega_{\varepsilon}\right)}\right) \\
& \leqslant \lambda^{-1} C\left(\varepsilon^{\frac{1+\sigma}{2}}+c(\delta)\left\|\Phi_{\varepsilon, \mathbf{P}}\right\|_{H^{2}\left(\Omega_{\varepsilon}\right)}\right)
\end{aligned}
$$

we have

$$
\left\|\Phi_{\varepsilon, \mathbf{P}}\right\|_{H^{2}\left(\Omega_{\varepsilon}\right)} \leqslant C \varepsilon^{\frac{1+\sigma}{2}}
$$

We have proved

LEMMA 3.4. - There exists $\bar{\varepsilon}>0$ such that for every $(N+1)$-tuple $\varepsilon, P_{1}, \ldots, P_{K}$ with $0<\varepsilon<\bar{\varepsilon}$ and $\mathbf{P}=\left(P_{1}, \ldots, P_{K}\right) \in \bar{\Lambda}$ there is a unique $\Phi_{\varepsilon, \mathbf{P}} \in \mathcal{K}_{\varepsilon, \mathbf{P}}^{\perp}$ satisfying $S_{\varepsilon}\left(\sum_{i=1}^{K} P w_{i}+\Phi_{\varepsilon, \mathbf{P}}\right) \in \mathcal{C}_{\varepsilon, \mathbf{P}}$ and

$$
\left\|\Phi_{\varepsilon, \mathbf{P}}\right\|_{H^{2}\left(\Omega_{\varepsilon}\right)} \leqslant C \varepsilon^{\frac{1+\sigma}{2}} .
$$

The next lemma is our main estimate.

LEMMA 3.5. - Let $\Phi_{\varepsilon, \mathbf{P}}$ be defined by Lemma 3.4. Then we have

$$
\begin{aligned}
J_{\varepsilon}\left(\sum_{i=1}^{K} P w_{i}+\Phi_{\varepsilon, \mathbf{P}}\right) \\
=\varepsilon^{N}\left[\frac{K}{2} I(w)-\gamma_{1} \varepsilon \sum_{i=1}^{K} H\left(P_{i}\right)\right. \\
\left.\quad-\frac{1}{2} \sum_{k, l=1, \ldots, K, k \neq l}\left(\gamma_{k l}+\mathrm{o}(1)\right) w\left(\frac{\left|P_{k}-P_{l}\right|}{\varepsilon}\right)+\mathrm{o}(\varepsilon)\right],
\end{aligned}
$$


where $\gamma_{1}$ and $\gamma_{k l}$ are defined in Lemma 2.5.

Proof. - In fact for any $\mathbf{P} \in \bar{\Lambda}$, we have

$$
\begin{aligned}
& \varepsilon^{-N} J_{\varepsilon}\left(\sum_{i=1}^{K} P w_{i}+\Phi_{\varepsilon, \mathbf{P}}\right) \\
& =\varepsilon^{-N} J_{\varepsilon}\left(\sum_{i=1}^{K} P w_{i}\right)+g_{\varepsilon, \mathbf{P}}\left(\Phi_{\varepsilon, \mathbf{P}}\right)+\mathrm{O}\left(\left\|\Phi_{\varepsilon, \mathbf{P}}\right\|_{H^{2}\left(\Omega_{\varepsilon}\right)}^{2}\right),
\end{aligned}
$$

where

$$
\begin{aligned}
& g_{\varepsilon, \mathbf{P}}\left(\Phi_{\varepsilon, \mathbf{P}}\right) \\
& \quad=\int_{\Omega_{\varepsilon}} \sum_{i=1}^{K}\left(\nabla P w_{i} \nabla \Phi_{\varepsilon, \mathbf{P}}+P w_{i} \Phi_{\varepsilon, \mathbf{P}}\right)-\int_{\Omega_{\varepsilon}} f\left(\sum_{i=1}^{K} P w_{i}\right) \Phi_{\varepsilon, \mathbf{P}} \\
& \quad=\int_{\Omega_{\varepsilon}}\left[\sum_{i=1}^{K} f\left(w_{i}\right)-f\left(\sum_{i=1}^{K} P w_{i}\right)\right] \Phi_{\varepsilon, \mathbf{P}} \\
& \quad \leqslant\left\|\sum_{i=1}^{K} f\left(w_{i}\right)-f\left(\sum_{i=1}^{K} P w_{i}\right)\right\|_{L^{2}}\left\|\Phi_{\varepsilon, \mathbf{P}}\right\|_{L^{2}\left(\Omega_{\varepsilon}\right)} \\
& \quad \leqslant \mathrm{O}\left(\varepsilon^{1+\sigma}\right)
\end{aligned}
$$

by Lemmas 3.3 and 3.4.

Estimate (3.19) now follows from Lemmas 2.6 and 3.4.

Finally, we show that $\Phi_{\varepsilon, \mathbf{P}}$ is actually smooth in $\mathbf{P}$.

LEMmA 3.6. - Let $\Phi_{\varepsilon, \mathbf{P}}$ be defined by Lemma 3.4. Then $\Phi_{\varepsilon, \mathbf{P}} \in C^{1}$ in $\mathbf{P}$.

Proof. - Recall that $\Phi_{\varepsilon, \mathbf{P}}$ is a solution of the equation

$$
\pi_{\varepsilon, \mathbf{P}} \circ S_{\varepsilon}\left(\sum_{i=1}^{K} P w_{i}+\Phi_{\varepsilon, \mathbf{P}}\right)=0
$$

such that

$$
\Phi_{\varepsilon, \mathbf{P}} \in \mathcal{K}_{\varepsilon, P}^{\perp} .
$$

By differentiating Eq. (3.19) twice we easily conclude that the functions $P w_{i}$ and $\partial^{2} P w_{i} /\left(\partial \tau_{P_{i, j}} \partial \tau_{P_{i, k}}\right)$ are $C^{1}$ in $\mathbf{P}$. This implies that the projection $\pi_{\varepsilon, \mathbf{P}}$ is $C^{1}$ in $\mathbf{P}$. Applying $\partial / \partial \tau_{P_{i, j}}$ gives 


$$
\begin{gathered}
\pi_{\varepsilon, \mathbf{P}} \circ D S_{\varepsilon}\left(\sum_{i=1}^{K} P w_{i}+\Phi_{\varepsilon, \mathbf{P}}\right)\left(\sum_{i=1}^{K} \frac{\partial P w_{i}}{\partial \tau_{P_{i, j}}}+\frac{\partial \Phi_{\varepsilon, \mathbf{P}}}{\partial \tau_{P_{i, j}}}\right) \\
+\frac{\partial \pi_{\varepsilon, \mathbf{P}}}{\partial \tau_{P_{i, j}}} \circ S_{\varepsilon}\left(\sum_{i=1}^{K} P w_{i}+\Phi_{\varepsilon, \mathbf{P}}\right)=0
\end{gathered}
$$

where

$$
D S_{\varepsilon}\left(\sum_{i=1}^{K} P w_{i}+\Phi_{\varepsilon, \mathbf{P}}\right)=\Delta-1+f^{\prime}\left(\sum_{i=1}^{K} P w_{i}+\Phi_{\varepsilon, \mathbf{P}}\right)
$$

We decompose $\partial \Phi_{\varepsilon, \mathbf{P}} / \partial \tau_{P_{i, j}}$ into two parts:

$$
\frac{\partial \Phi_{\varepsilon, \mathbf{P}}}{\partial \tau_{P_{i, j}}}=\left(\frac{\partial \Phi_{\varepsilon, \mathbf{P}}}{\partial \tau_{P_{i, j}}}\right)_{1}+\left(\frac{\partial \Phi_{\varepsilon, \mathbf{P}}}{\partial \tau_{P_{i, j}}}\right)_{2}
$$

where $\left(\partial \Phi_{\varepsilon, \mathbf{P}} / \partial \tau_{P_{i, j}}\right)_{1} \in \mathcal{K}_{\varepsilon, \mathbf{P}}$ and $\left(\partial \Phi_{\varepsilon, \mathbf{P}} / \partial \tau_{P_{i, j}}\right)_{2} \in \mathcal{K}_{\varepsilon, \mathbf{P}}^{\perp}$.

We can easily show that $\left(\partial \Phi_{\varepsilon, \mathbf{P}} / \partial \tau_{P_{i, j}}\right)_{1}$ is continuous in $\mathbf{P}$ since

$$
\int_{\Omega_{\varepsilon}} \Phi_{\varepsilon, \mathbf{P}} \frac{\partial P w_{k}}{\partial \tau_{P_{k, l}}}=0, \quad k=1, \ldots, K, l=1, \ldots, N-1,
$$

and

$$
\begin{gathered}
\int_{\Omega_{\varepsilon}} \frac{\partial \Phi_{\varepsilon, \mathbf{P}}}{\partial \tau_{P_{i, j}}} \frac{\partial P w_{k}}{\partial \tau_{P_{k, l}}}+\int_{\Omega_{\varepsilon}} \Phi_{\varepsilon, \mathbf{P}} \frac{\partial^{2} P w_{k}}{\partial \tau_{P_{i, j}} \partial \tau_{P_{k, l}}}=0, \\
k, i=1, \ldots, K, \quad l, j=1, \ldots, N-1 .
\end{gathered}
$$

We can write Eq. (3.23) as

$$
\begin{aligned}
& \pi_{\varepsilon, \mathbf{P}} \circ D S_{\varepsilon}\left(\sum_{i=1}^{K} P w_{i}+\Phi_{\varepsilon, \mathbf{P}}\right)\left(\left(\frac{\partial \Phi_{\varepsilon, \mathbf{P}}}{\partial \tau_{P_{i, j}}}\right)_{2}\right) \\
& +\pi_{\varepsilon, \mathbf{P}} \circ D S_{\varepsilon}\left(\sum_{i=1}^{K} P w_{i}+\Phi_{\varepsilon, \mathbf{P}}\right)\left(\sum_{i=1}^{K} \frac{\partial P w_{i}}{\partial \tau_{P_{i, j}}}+\left(\frac{\partial \Phi_{\varepsilon, \mathbf{P}}}{\partial \tau_{P_{i, j}}}\right)_{1}\right) \\
& +\frac{\partial \pi_{\varepsilon, \mathbf{P}}}{\partial \tau_{P_{i, j}}} \circ S_{\varepsilon}\left(\sum_{i=1}^{K} P w_{i}+\Phi_{\varepsilon, \mathbf{P}}\right)=0 .
\end{aligned}
$$


As in the proof of Propositions 3.1 and 3.2, we can show that the operator

$$
\pi_{\varepsilon, \mathbf{P}} \circ D S_{\varepsilon}\left(\sum_{i=1}^{K} P w_{i}+\Phi_{\varepsilon, \mathbf{P}}\right)
$$

is invertible from $\mathcal{K}_{\varepsilon, \mathbf{P}}^{\perp}$ to $\mathcal{C}_{\varepsilon, \mathbf{P}}^{\perp}$. Then we can take inverse of $\pi_{\varepsilon, \mathbf{P}} \circ$ $D S_{\varepsilon}\left(\sum_{i=1}^{K} P w_{i}+\Phi_{\varepsilon, \mathbf{P}}\right)$ in the above equation and the inverse is continuous in $\mathbf{P}$.

Since $\partial P w_{i} / \partial \tau_{P_{i, j}},\left(\partial \Phi_{\varepsilon, \mathbf{P}} / \partial \tau_{P_{i, j}}\right)_{1} \in \mathcal{K}_{\varepsilon, \mathbf{P}}$ are continuous in $\mathbf{P}$ and so is $\partial \pi_{\varepsilon, \mathbf{P}} / \partial \tau_{P_{i, j}}$, we conclude that $\left(\partial \Phi_{\varepsilon, \mathbf{P}} / \partial \tau_{P_{i, j}}\right)_{2}$ is also continuous in $\mathbf{P}$. This is the same as the $C^{1}$ dependence of $\Phi_{\varepsilon, \mathbf{P}}$ in $\mathbf{P}$. The proof is finished.

\section{THE REDUCED PROBLEM: A MAXIMIZING PROCEDURE}

In this section, we study a maximizing problem.

Fix $\mathbf{P} \in \bar{\Lambda}$. Let $\Phi_{\varepsilon, \mathbf{P}}$ be the solution given by Lemma 3.4. We define a new functional

$$
M_{\varepsilon}(\mathbf{P})=J_{\varepsilon}\left(\sum_{i=1}^{K} P w_{i}+\Phi_{\varepsilon, \mathbf{P}}\right): \bar{\Lambda} \rightarrow R .
$$

We shall prove

PROPOSITION 4.1. - For $\varepsilon$ small, the following maximizing problem

$$
\max \left\{M_{\varepsilon}(\mathbf{P}): \mathbf{P} \in \bar{\Lambda}\right\}
$$

has a solution $\mathbf{P}^{\varepsilon} \in \Lambda$.

Proof. - Since $J_{\varepsilon}\left(\sum_{i=1}^{K} P_{\Omega_{\varepsilon}, P_{i}} w+\Phi_{\varepsilon, \mathbf{P}}\right)$ is continuous in $\mathbf{P}$, the maximizing problem has a solution. Let $M_{\varepsilon}\left(\mathbf{P}^{\varepsilon}\right)$ be the maximum where $\mathbf{P}^{\varepsilon} \in \bar{\Lambda}$.

We claim that $\mathbf{P}^{\varepsilon} \in \Lambda$.

In fact for any $\mathbf{P} \in \bar{\Lambda}$, by Lemma 3.5, we have

$$
\begin{aligned}
M_{\varepsilon}(\mathbf{P})= & \varepsilon^{N}\left[\frac{K}{2} I(w)-\varepsilon \gamma\left(\sum_{i=1}^{K} H\left(P_{i}\right)\right)\right. \\
& \left.-\frac{1}{2} \sum_{k, l=1, \ldots, K, k \neq l}\left(\gamma_{k l}+o(1)\right) w\left(\frac{\left|P_{k}-P_{l}\right|}{\varepsilon}\right)+o(\varepsilon)\right] .
\end{aligned}
$$


Since $M_{\varepsilon}(\mathbf{P})^{\varepsilon}$ is the maximum, we have

$$
\begin{aligned}
& \gamma_{1} \sum_{i=1}^{K} H\left(P_{i}^{\varepsilon}\right)+\frac{1}{\varepsilon} \sum_{k \neq l}\left(\frac{1}{2} \gamma_{k l}+\mathrm{o}(1)\right) w\left(\frac{\left|P_{k}^{\varepsilon}-P_{l}^{\varepsilon}\right|}{\varepsilon}\right) \\
& \leqslant \gamma_{1} \sum_{i=1}^{K} H\left(P_{i}\right)+\frac{1}{\varepsilon} \sum_{k \neq l}\left(\frac{1}{2} \gamma_{k l}+\mathrm{o}(1)\right) w\left(\frac{\left|P_{k}-P_{l}\right|}{\varepsilon}\right)+\mathrm{o}(1)
\end{aligned}
$$

for any $\mathbf{P}=\left(P_{1}, \ldots, P_{K}\right) \in \bar{\Lambda}$.

Choose $P_{i}$ such that $H\left(P_{i}\right) \rightarrow \min _{P \in \Gamma_{i}} H(P)$ and $w\left(\left|P_{k}-P_{l}\right| / \varepsilon\right) / \varepsilon \rightarrow$ 0 . This implies that

$$
\begin{aligned}
& \gamma_{1} \sum_{i=1}^{K} H\left(P_{i}^{\varepsilon}\right)+\frac{1}{\varepsilon} \sum_{k \neq l}\left(\frac{1}{2} \gamma_{k l}+o(1)\right) w\left(\frac{\left|P_{k}^{\varepsilon}-P_{l}^{\varepsilon}\right|}{\varepsilon}\right) \\
& \quad \leqslant \gamma_{1} \sum_{i=1}^{K} \min _{P \in \Gamma_{i}} H(P)+\delta
\end{aligned}
$$

for any $\delta>0$.

Note that $\partial \Lambda \subset\left\{P_{i} \in \partial \Gamma_{i}\right.$ or $\left.w\left(\left|P_{k}-P_{l}\right| / \varepsilon\right)=\varepsilon \eta\right\}$. Hence if $\mathbf{P} \in \partial \Lambda$, we have that either

$$
H\left(P_{i}\right) \geqslant \min _{P \in \partial \Gamma_{i}} H(P) \geqslant \min _{P \in \Gamma_{i}} H(P)+2 \eta_{0}
$$

for some $i=1, \ldots, K$ and $\eta_{0}>0$ (by condition (1.5)), or

$$
\frac{1}{\varepsilon} w\left(\frac{\left|P_{k}-P_{l}\right|}{\varepsilon}\right)=\eta
$$

for some $k \neq l$.

Hence if $\mathbf{P} \in \partial \Lambda$ we have

$$
\begin{aligned}
& \gamma_{1} \sum_{i=1}^{K} H\left(P_{i}^{\varepsilon}\right)+\frac{1}{\varepsilon} \sum_{k \neq l}\left(\frac{1}{2} \gamma_{k l}+o(1)\right) w\left(\frac{\left|P_{k}^{\varepsilon}-P_{l}^{\varepsilon}\right|}{\varepsilon}\right) \\
& \geqslant \gamma_{1} \sum_{i=1}^{K} \min _{P \in \Gamma_{i}} H(P)+\min \left(\gamma_{1} \eta_{0}, \min _{k \neq l, w\left(\left|P_{k}-P_{l}\right| / \varepsilon\right)=\eta \varepsilon} \gamma_{k l} \eta\right) .
\end{aligned}
$$

Note that

$$
\min _{k \neq l, w\left(\left|P_{k}-P_{l}\right| / \varepsilon\right)=\eta \varepsilon} \gamma_{k l} \geqslant \inf _{\tau \in \Sigma_{1}} \tau \geqslant \delta_{0}>0
$$


since for any $\tau \in \Sigma_{1}$, we have

$$
\tau=\int_{R_{+}^{N}} f(w) \mathrm{e}^{\langle b, y\rangle}=\frac{1}{2} \int_{R^{N}} f(w) \mathrm{e}^{\langle b, y\rangle}>0 .
$$

A contradiction to (4.3) if we choose $\delta$ small enough.

It follows that $\mathbf{P}^{\varepsilon} \in \Lambda$.

This completes the proof of Proposition 4.1.

\section{PROOF OF THEOREM 1.2}

In this section section, we apply results in Sections 3 and 4 to prove Theorems 1.1, 1.2 and Corollary 1.3.

Proofs of Theorems 1.1, 1.2 and Corollary 1.3. - By Lemmas 3.4 and 3.6, there exists $\varepsilon_{0}$ such that for $\varepsilon<\varepsilon_{0}$ we have a $C^{1}$ map which, to any $\mathbf{P} \in \bar{\Lambda}$, associates $\Phi_{\varepsilon, \mathbf{P}} \in \mathcal{K}_{\varepsilon, \mathbf{P}}^{\perp}$ such that

$$
S_{\varepsilon}\left(\sum_{i=1}^{K} P w_{i}+\Phi_{\varepsilon, \mathbf{P}}\right)=\sum_{k=1, \ldots, K ; l=1, \ldots, N-1} \alpha_{k l} \frac{\partial P w_{k}}{\partial \tau_{P_{k, l}}}
$$

for some constants $\alpha_{k l} \in R^{K(N-1)}$.

By Proposition 4.1, we have $\mathbf{P}^{\varepsilon} \in \Lambda$, achieving the maximum of the maximization problem in Proposition 4.1. Let $\Phi_{\varepsilon}=\Phi_{\varepsilon, \mathbf{P}^{\varepsilon}}$ and $u_{\varepsilon}=$ $\sum_{i=1}^{K} P_{\Omega_{\varepsilon, P_{i}^{\varepsilon}}} w+\Phi_{\varepsilon, \mathbf{P}^{\varepsilon}}$. Then we have

$$
\left.\frac{\partial}{\partial \tau_{P_{i, j}}}\right|_{\mathbf{P}=\mathbf{P}^{\varepsilon}} M_{\varepsilon}\left(\mathbf{P}^{\varepsilon}\right)=0, \quad i=1, \ldots, K, j=1, \ldots, N-1 .
$$

Hence we have

$$
\begin{aligned}
& \int_{\Omega_{\varepsilon}}\left[\left.\nabla u_{\varepsilon} \nabla \frac{\partial\left(\sum_{i=1}^{K} P w_{i}+\Phi_{\varepsilon, \mathbf{P}}\right)}{\partial \tau_{P_{i, j}}}\right|_{\mathbf{P}=\mathbf{P}^{\varepsilon}}+\left.u_{\varepsilon} \frac{\partial\left(\sum_{i=1}^{K} P w_{i}+\Phi_{\varepsilon, \mathbf{P}}\right)}{\partial \tau_{P_{i, j}}}\right|_{\mathbf{P}=\mathbf{P}^{\varepsilon}}\right. \\
& \left.\quad-\left.f\left(u_{\varepsilon}\right) \frac{\partial\left(\sum_{i=1}^{K} P w_{i}+\Phi_{\varepsilon, \mathbf{P}}\right)}{\partial \tau_{P_{i, j}}}\right|_{\mathbf{P}=\mathbf{P}^{\varepsilon}}\right]=0 .
\end{aligned}
$$

Thus

$$
\left.\int_{\Omega_{\varepsilon}} \nabla u_{\varepsilon} \nabla \frac{\partial\left(P w_{i}+\Phi_{\varepsilon, \mathbf{P}}\right)}{\partial \tau_{P_{i, j}}}\right|_{\mathbf{P}=\mathbf{P}^{\varepsilon}}+\left.u_{\varepsilon} \frac{\partial\left(P w_{i}+\Phi_{\varepsilon, \mathbf{P}}\right)}{\partial \tau_{P_{i, j}}}\right|_{\mathbf{P}=\mathbf{P}^{\varepsilon}}
$$




$$
-\left.f\left(u_{\varepsilon}\right) \frac{\partial\left(P w_{i}+\Phi_{\varepsilon, \mathbf{P}}\right)}{\partial \tau_{P_{i, j}}}\right|_{\mathbf{P}=\mathbf{P}^{\varepsilon}}=0
$$

for $i=1, \ldots, K$ and $j=1, \ldots, N-1$.

Therefore we have

$$
\sum_{k=1, \ldots, K ; l=1, \ldots, N-1} \alpha_{k l} \int_{\Omega_{\varepsilon}} \frac{\partial P w_{k}}{\partial \tau_{P_{k, l}}} \frac{\partial\left(P w_{i}+\Phi_{\varepsilon, \mathbf{P}}\right)}{\partial \tau_{P_{i, j}}}=0 .
$$

Since $\Phi_{\varepsilon, \mathbf{P}} \in \mathcal{K}_{\varepsilon, \mathbf{P}}^{\perp}$, we have that

$$
\begin{aligned}
\left|\int_{\Omega_{\varepsilon}} \frac{\partial P w_{k}}{\partial \tau_{P_{k, l}}} \frac{\partial \Phi_{\varepsilon, \mathbf{P}}}{\partial \tau_{P_{i, j}}}\right| & =\left|-\int_{\Omega_{\varepsilon}} \frac{\partial^{2} P w_{i}}{\partial \tau_{P_{k, l}} \partial \tau_{P_{i, j}}} \Phi_{\varepsilon, \mathbf{P}}\right| \\
& \leqslant\left\|\frac{\partial^{2} P w_{i}}{\partial \tau_{P_{k, l}} \partial \tau_{P_{i, j}}}\right\|_{L^{2}}\left\|\Phi_{\varepsilon, \mathbf{P}}\right\|_{L^{2}} \\
& =\mathrm{O}\left(\varepsilon^{-2+\frac{1+\sigma}{2}}\right) .
\end{aligned}
$$

Note that

$$
\int_{\Omega_{\varepsilon}} \frac{\partial P w_{k}}{\partial \tau_{P_{k, l}}} \frac{\partial P w_{i}}{\partial \tau_{P_{i, j}}}=\frac{1}{\varepsilon^{2}} \delta_{i k} \delta_{l j}(A+\mathrm{o}(1)),
$$

where

$$
A=\int_{R_{+}^{N}}\left(\frac{\partial w}{\partial y_{1}}\right)^{2}>0
$$

Thus Eq. (5.2) becomes a system of homogeneous equations for $\alpha_{k l}$ and the matrix of the system is nonsingular since it is diagonally dominant. So $\alpha_{k l} \equiv 0, k=1, \ldots, K, l=1, \ldots N-1$.

Hence $u_{\varepsilon}=\sum_{i=1}^{K} P_{\Omega_{\varepsilon, P_{i}^{\varepsilon}}} w+\Phi_{\varepsilon, P_{1}^{\varepsilon}, \ldots, P_{K}^{\varepsilon}}$ is a solution of (1.2).

By our construction, it is easy to see that by the maximum principle $u_{\varepsilon}>0$ in $\Omega$. Moreover $\varepsilon^{N} J_{\varepsilon}\left(u_{\varepsilon}\right) \rightarrow(K / 2) I(w)$ and $u_{\varepsilon}$ has only $K$ local maximum points $Q_{1}^{\varepsilon}, \ldots, Q_{K}^{\varepsilon}$ and $Q_{i}^{\varepsilon} \in \partial \Omega$. By the structure of $u_{\varepsilon}$ we see that (up to a permutation) $Q_{i}^{\varepsilon}-P_{i}^{\varepsilon}=\mathrm{o}(1)$. This proves Theorem 1.2.

Theorem 1.1 follows from Theorem 1.2 by taking $\Gamma_{i}=\Gamma, i=1$, $\ldots, K$.

Finally, we prove Corollary 1.3.

If $\Omega$ is not a ball, then $H(P)$ has a local minimum on some open set $\Gamma$, Theorem 1.1 can be applied. 
If $\Omega$ is a ball, Corollary 1.3 follows by minimizing energy in symmetric spaces. See [21] and [23].

\section{ACKNOWLEDGEMENT}

The research of the first author is supported by a NSERC grant of Canada. The research of the second author is supported by an Earmarked Grant from RGC of Hong Kong.

\section{REFERENCES}

[1] G. Adimurthi Mancinni and S.L. Yadava, The role of mean curvature in a semilinear Neumann problem involving the critical Sobolev exponent, Comm. P.D.E. 20 (1995) 591-631.

[2] F. Adimurthi PACELla and S.L. Yadava, Interaction between the geometry of the boundary and positive solutions of a semilinear Neumann problem with critical nonlinearity, J. Funct. Anal. 113 (1993) 318-350.

[3] F. Adimurthi Pacella and S.L. Yadava, Characterization of concentration points and $L^{\infty}$-estimates for solutions involving the critical Sobolev exponent, Differential Integral Equations 8 (1) (1995) 41-68.

[4] S. Agmon, Lectures on Elliptic Boundary Value Problems, Van Nostrand, Princeton, 1965.

[5] D.G. Aronson and H.F. Weinberger, Multidimensional nonlinear diffusion arising in population genetics, Adv. in Math. 30 (1978) 33-76.

[6] E.N. DANCER, A note on asymptotic uniqueness for some nonlinearities which change sign, Rocky Mountain Math. J., to appear.

[7] A. Floer and A. Weinstein, Nonspreading wave packets for the cubic Schrödinger equation with a bounded potential, J. Funct. Anal. 69 (1986) 397-408.

[8] R. Gardner and L.A. Peletier, The set of positive solutions of semilinear equations in large balls, Proc. Roy. Soc. Edinburgh 104 A (1986) 53-72.

[9] B. Gidas, W.-M. NI, and L. NiREnberG, Symmetry of positive solutions of nonlinear elliptic equations in $R^{n}$, in: Mathematical Analysis and Applications, Part A, Adv. Math. Suppl. Studies, Vol. 7A, Academic Press, New York, 1981, pp. 369-402.

[10] D. Gilbarg and N.S. Trudinger, Elliptic Partial Differential Equations of Second Order, 2nd ed., Springer, Berlin, 1983.

[11] C. GuI, Multi-peak solutions for a semilinear Neumann problem, Duke Math. J. 84 (1996) 739-769.

[12] C. Gui and N. Ghoussoub, Multi-peak solutions for a semilinear Neumann problem involving the critical Sobolev exponent, Math. Z. 229 (1998) 443-474.

[13] C. GUI and J. WEI, Multiple interior peak solutions for some singularly perturbed Neumann problems, J. Differential Equations 158 (1999) 1-27.

[14] B. HELFFER and J. SJÖSTRAND, Multiple wells in the semi-classical limit I, Comm. P.D.E. 9 (1984) 337-408. 
[15] J. JANG, On spike solutions of singularly perturbed semilinear Dirichlet problems, J. Differential Equations 114 (1994) 370-395.

[16] M.K. KWONG, Uniqueness of positive solutions of $\Delta u-u+u^{p}=0$ in $R^{n}$, Arch. Rational Mech. Anal. 105 (1989) 243-266.

[17] Y.Y. LI, On a singularly perturbed equation with Neumann boundary condition, Comm. P.D.E. 23 (1998) 487-545.

[18] C. Lin, W.-M. Ni and I. TAKAgI, Large amplitude stationary solutions to a chemotaxis systems, J. Differential Equations 72 (1988) 1-27.

[19] J.L. Lions and E. MAgenes, Non-Homogeneous Boundary Value Problems and Applications, Vol I, Springer, New York, Berlin, Heidelberg, Tokyo, 1972.

[20] W.-M. NI, X. PAN and I. TAKAGI, Singular behavior of least-energy solutions of a semilinear Neumann problem involving critical Sobolev exponents, Duke Math. J. 67 (1992) 1-20.

[21] W.-M. Ni and I. TAKAGI, On the shape of least energy solutions to a semilinear Neumann problem, Comm. Pure Appl. Math. 41 (1991) 819-851.

[22] W.-M. NI and I. TAKAGI, Locating the peaks of least energy solutions to a semilinear Neumann problem, Duke Math. J. 70 (1993) 247-281.

[23] W.-M. Ni and I. TAKagi, Point-condensation generated by a reaction-diffusion system in axially symmetric domains, Japan J. Industrial Appl. Math. 12 (1995) 327-365.

[24] W.-M. NI and J. WEI, On the location and profile of spike-layer solutions to singularly perturbed semilinear Dirichlet problems, Comm. Pure Appl. Math. 48 (1995) 731-768.

[25] Y.G. OH, Existence of semi-classical bound states of nonlinear Schrödinger equations with potentials of the class $(V)_{a}$, Comm. P.D.E. 13 (12) (1988) 14991519.

[26] Y.G. ОH, On positive multi-lump bound states of nonlinear Schrödinger equations under multiple-well potentials, Comm. Math. Phys. 131 (1990) 223-253.

[27] X.B. PAN, Condensation of least-energy solutions of a semilinear Neumann problem, J. Partial Differential Equations 8 (1995) 1-36.

[28] X.B. PAN, Condensation of least-energy solutions: the effect of boundary conditions, Nonlinear Analysis, TMA 24 (1995) 195-222.

[29] X.B. PAN, Further study on the effect of boundary conditions, J. Differential Equations 117 (1995) 446-468.

[30] J. SMOller and A. WASSERMAn, Global bifurcation of steady-state solutions, J. Differential Equations 39 (1981) 269-290.

[31] Z.-Q. WANG, On the existence of multiple single-peaked solutions for a semilinear Neumann problem, Arch. Rational Mech. Anal. 120 (1992) 375-399.

[32] M. WARD, An asymptotic analysis of localized solutions for some reaction-diffusion models in multidimensional domains, Stud. Appl. Math. 97 (1996) 103-126.

[33] J. WEI, On the construction of single-peaked solutions of a singularly perturbed semilinear Dirichlet problem, J. Differential Equations 129 (1996) 315-333.

[34] J. WEI, On the effect of the geometry of the domain in a singularly perturbed Dirichlet problem, Differential Integral Equations, to appear.

[35] J. WEI, On the boundary spike layer solutions of singularly perturbed semilinear Neumann problem, J. Differential Equations 134 (1997) 104-133. 
[36] J. WEI, On the construction of single interior peak solutions for a singularly perturbed Neumann problem, in: Partial Differential Equations: Theory and Numerical solution; CRC Press LLC, 1998, pp. 336-349.

[37] J. WEI and M. WinTER, Stationary solutions for the Cahn-Hilliard equation, Ann. Inst. H. Poincaré Anal. Non Linéaire 15 (1998) 459-492.

[38] J. WEI and M. WinTER, Multiple boundary spike solutions for a wide class of singular perturbation problems, J. London Math. Soc. 59 (2) (1999) 585-606.

[39] K. YosidA, Functional Analysis, 5th ed., Springer, Berlin, 1978.

[40] E. ZeIdLer, Nonlinear Functional Analysis and its Applications I, Fixed-Point Theorems, Springer, Berlin, 1986. 